Issued by Sandia National Laboratories, operated for the United States Department of Energy by Sandia Corporation.

NOTICE: This report was prepared as an account of work sponsored by an agency of the United States Government. Neither the United States Government, nor any agency thereof, nor any of their employees, nor any of their contractors, subcontractors, or their employees, make any warranty, express or implied, or assume any legal liability or responsibility for the accuracy, completeness, or usefulness of any information, apparatus, product, or process disclosed, or represent that its use would not infringe privately owned rights. Reference herein to any specific commercial product, process, or service by trade name, trademark, manufacturer, or otherwise, does not necessarily constitute or imply its endorsement, recommendation, or favoring by the United States Government, any agency thereof, or any of their contractors or subcontractors. The views and opinions expressed herein do not necessarily state or reflect those of the United States Government, any agency thereof, or any of their contractors.

Printed in the United States of America. This report has been reproduced directly from the best available copy.

Available to DOE and DOE contractors from Office of Scientific and Technical Information

P.O. Box 62

Oak Ridge, TN 37831

Prices available from (703) 605-6000

Web site: http://www.ntis.gov/ordering.htm

Available to the public from

National Technical Information Service

U.S. Department of Commerce

5285 Port Royal Rd

Springfield, VA 22161

NTIS price codes

Printed copy: A03

Microfiche copy: A01

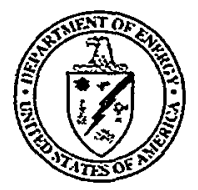




\section{DISCLAIMER}

Portions of this document may be illegible in electronic image products. Images are produced from the best available original document. 
SAND99-2901

Unlimited Release

Printed November 1999

\title{
Portable Imaging Polarimeter and Imaging Experiments
}

\author{
G.S. Phipps \\ Optics and Emerging Technology \\ S.A. Kemme and W.C. Sweatt \\ Photonics Research \\ J. R. Wendt and G. A. Vawter \\ Semiconductor Materials and Processes \\ Sandia National Laboratories \\ PO Box 5800 \\ Albuquerque, NM 87185-0980
M.R. Descour, D.S. Sabatke, J.P. Garcia and E.L. Dereniak
Optical Sciences Center,
University of Arizona, Tucson, AZ 85721

\begin{abstract}
Polarimetry is the method of recording the state of polarization of light. Imaging polarimetry extends this method to recording the spatially resolved state of polarization within a scene. Imaging-polarimetry data have the potential to improve the detection of manmade objects in natural backgrounds. We have constructed a midwave infrared complete imaging polarimeter consisting of a fixed wire-grid polarizer and rotating form-birefringent retarder. The retardance and the orientation angles of the retarder were optimized to minimize the sensitivity of the instrument to noise in the measurements. The optimal retardance was found to be $132^{\circ}$ rather than the typical $90^{\circ}$. The complete imaging polarimeter utilized a liquid-nitrogen cooled PtSi camera. The fixed wire-grid polarizer was located at the cold stop inside the camera dewar. The complete imaging polarimeter was operated in the 4.42-5 $\mu \mathrm{m}$ spectral range. A series of imaging experiments was performed using as targets a surface of water, an automobile, and an aircraft. Further analysis of the polarization measurements revealed that in all three cases the magnitude of circular polarization was comparable to the noise in the calculated Stokes-vector components.
\end{abstract}




\section{Acknowledgements}

The authors are grateful to John Lamonica for arranging access to the Cessna aircraft depicted in this report. The results presented in this report were made possible through the efforts of Michael Descour, Derek Sabatke, John Garcia, and Eustace Dereniak, all of the Optical Sciences Center at the University of Arizona. 


\section{Contents}

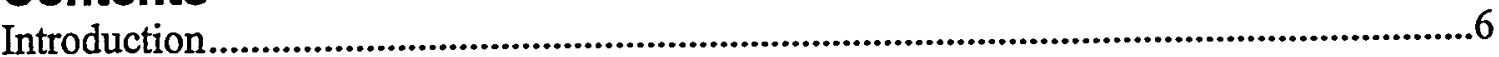

Design of Complete Polarimeter.........................................................................................

Selection of Retardance and Retarder Orientation Angles ........................................8

Tolerance to Variation of Retardance

with Wavelength and Angle of Incidence...................................................................10

Interpretation of Optimal Complete Polarimeter Design

Using The Poincare Sphere..................................................................................................12

Design and Fabrication of the Retarder Plate ........................................................................14

Characterization of Polarimeter Components .....................................................................16

Characterization Results ..........................................................................................17

Wire Grid Polarizer Characterization ...........................................................17

Form-birefringent Retarder Characterization .............................................17

Complete Imaging Polarimeter ...........................................................................................22

Experimental Results ......................................................................................................24

Outdoor Experiments: Idling Automobile ................................................................24

Aircraft.

Conclusions and Future Work ..........................................................................................29

References.

APPENDIX A. Poincare Sphere Cutout...........................................................................32

APPENDIX B. Virtual Instrument Program to Control the Characterization Set-up.........35

\section{Figures}

1 Surface and Contour Plots .......................................................................................11

2 Representation of the Poincare Sphere ...........................................................................12

3 Tetrahedron for a Configuration Reported by Ambirajan and Look.............................13

4 Simulated Waveplate Retardance Angle ........................................................................14

5 SEM Picture of the Rectangular Profile Grating as Fabricated......................................15

6 Simulated Waveplate Retardance Angle ........................................................................15

7 Polarizer Characterization Set-up ....................................................................................16

8 Virtual Instrument Interface '.......................................................................................17

9 Experimental Data ..................................................................................................19

10 Experimental Set-up .................................................................................................20

11 Retarder-Characterization Data ..................................................................................21

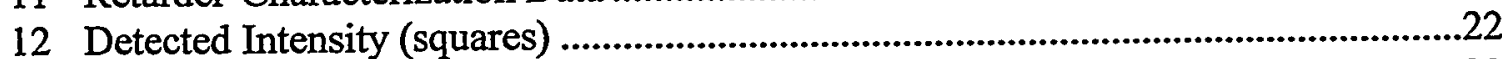

13 Measured Waveplate Retardances (squares) ................................................................22

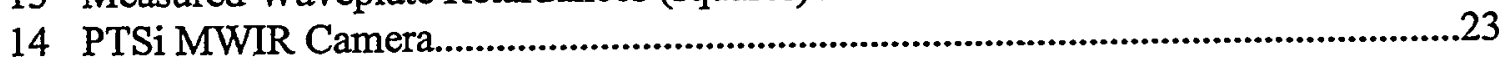

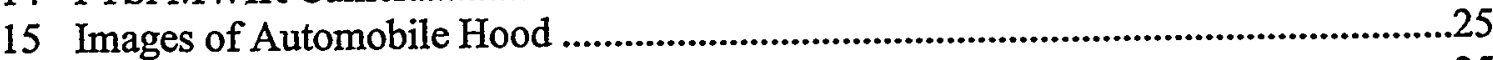

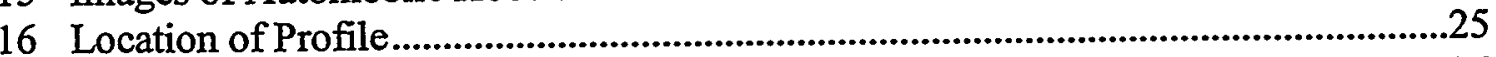

17 Profiles Through Automobile Image ............................................................................26

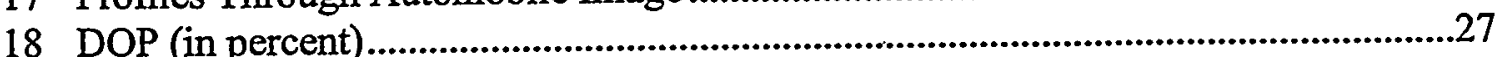

19 Stokes-parameter Images.......................................................................................28

20 Location of Profile Through Aircraft Image .................................................................29

21 DOP For Profile Indicated in Figure 20(a) ................................................................29

22 Wedged Double Wollaston Prism .....................................................................................31

23 Sequence in Which Four Polarization Images are Created ............................................31 


\section{INTRODUCTION}

The science of measuring the poiarization state of light is called polarimetry. There are two types of polarimeters: (1) those that actively irradiate an object with light of known polarization and (2) those that passively record radiation emitted and/or reflected by objects. Polarimeters in the thermal infrared [midwave infrared (MWIR)] are just becoming available, and accuracy and calibration issues are yet to be determined experimentally. The polarization effects of the instrument that measures light must be fully known, as well as the intervening atmosphere or media. In each case, a Mueller matrix can be used to describe instrumental and other effects. In addition, the polarimeter must be able to determine all Stokes parameters of the radiation at the instrument's entrance pupil. Such a polarimeter is known as a "complete" polarimeter. The subject of this report is the construction and experiments with a complete imaging polarimeter.

Stokes parameters can be used to describe light that is partially polarized. The first Stokes parameter, typically labeled $S_{0}$, represents the total irradiance recorded from an instantaneous field of view. The second Stokes parameter, $S_{1}$, represents the preference of the collected radiation for horizontal versus vertical polarization. A positive value of $S_{1}$ indicates horizontal polarization. The third Stokes parameter, $S_{2}$, represents the preference of the collected radiation for linear polarization oriented along $45^{\circ}$ and $135^{\circ}$ measured with respect to the horizontal direction. A positive value of $S_{2}$ indicates $45^{\circ}$ linear polarization. The fourth Stokes parameter, $S_{3}$, represents the preference for right-circular and left-circular polarization. A positive value of $S_{3}$ indicates right-circular polarization.

Polarimeters can be also divided into two classes: (1) time-sequential or (2) snapshot. The time-sequential polarimeter measures Stokes parameters by employing one or more rotating polarization elements. A snapshot polarimeter measures Stokes parameters by aperture or amplitude division. All parameters are measured simultaneously. In this report, we describe a time-sequential imaging polarimeter that employs a rotating form-birefringent retarder element.

The extension of polarimetry from the visible to the MWIR and long-wave infrared (LWIR) has been limited by the lack of IR polarizers and retarders. Also, in analogy to spectral imaging, the relatively recent availability of large-format MWIR imaging arrays is enabling an exploration of polarization's value in target and anomaly detection.

\section{Analogies with Spectral Imaging}

The retrieval of the four Stokes parameters at each pixel and subsequent data exploitation using these parameters is equivalent to recording and exploiting spectral irradiance at the focal plane of an imaging spectrometer. Instrumental calibration, in the form of a Mueller matrix or spectral transmittance, yields the Stokes parameters or spectral radiance at the instrument entrance pupil, respectively. Materials in the visible and nearinfrared (VNIR) and short-wave infrared (SWIR) can be usefully characterized by their spectral reflectance, detailed bi-directional reflectance distribution function (BRDF) effects being usually neglected. In the case of MWIR imaging polarimetry, targets need to be characterized by means of a $4 \times 4$ Mueller matrix. 
The main difference between spectral reflectance properties of a material and a Mueller matrix of a material is: a material does not convert spectral irradiance in one band into spectral radiance in another upon reflection. ${ }^{1}$ On the other hand, a material can turn one type of polarization into another. For example, a quarter-wave plate converts circular polarization into linear polarization.

\section{A Simple Complete Polarimeter Example}

The simplest polarimeter consists of a rotating quarter-wave plate and a fixed linear polarizer. The orientation of the quarter-wave plate's fast (or slow) axis is described by the rotation angle $\theta$. The fixed linear polarizer is located in the plane of the cold stop inside an infrared-camera dewar. Both polarimeter components, i.e., the quarter-wave plate and the linear polarizer, can be described by Mueller matrices (see Table 1).

\begin{tabular}{lcccc}
\hline \multicolumn{1}{c}{ Element Description } & Mueller Matrix \\
\hline Rotating quarter-wave plate & {$\left[\begin{array}{ccccc}1 & 0 & 0 & 0 \\
0 & \cos ^{2} 2 \theta & \cos 2 \theta \sin 2 \theta & -\sin 2 \theta \\
0 & \cos 2 \theta \sin 2 \theta & \sin ^{2} 2 \theta & \cos 2 \theta \\
0 & \sin 2 \theta & -\cos 2 \theta & 0\end{array}\right]$} \\
\hline Fixed linear polarizer & {$\left[\begin{array}{cccc}\frac{1}{2} & \frac{1}{2} & 0 & 0 \\
\frac{1}{2} & \frac{1}{2} & 0 & 0 \\
0 & 0 & 0 & 0 \\
0 & 0 & 0 & 0\end{array}\right]$} \\
\hline
\end{tabular}

Table 1. Mueller matrices used in the description of the simple polarimeter.

With this polarimeter, four measurements need to be collected to determine the four Stokes parameters. For example, the quarter wave plate is rotated to $\theta=0^{\circ}, 22.5^{\circ}, 45^{\circ}$, and $135^{\circ}$. The axis of the polarizer is oriented along $0^{\circ}$. The four detector-output measurements (for example, in volts, $V_{1}$ to $V_{4}$ ) and organized as a vector $\bar{V}$ are related to the four Stokes parameters, $S_{0}$ to $S_{3}$, through

$$
\left[\begin{array}{l}
V_{1} \\
V_{2} \\
V_{3} \\
V_{4}
\end{array}\right]=\left[\begin{array}{cccc}
\frac{1}{2} & \frac{1}{2} & 0 & 0 \\
\frac{1}{2} & 0 & 0 & \frac{1}{2} \\
\frac{1}{2} & 0 & 0 & -\frac{1}{2} \\
\frac{1}{2} & \frac{1}{4} & \frac{1}{4} & \frac{1}{2 \sqrt{2}}
\end{array}\right]\left[\begin{array}{l}
S_{0} \\
S_{1} \\
S_{2} \\
S_{3}
\end{array}\right]
$$

\footnotetext{
${ }^{1}$ An exception to this statement is the case of fluorescence but that is generally outside the scope of VNIR and SWIR remote-sensing applications.
} 
A discussion of the origin of this matrix-vector relation and the selection of optimal retarderorientation angles $\theta$ is contained in this report in the section Selection of Retardance and Retarder Orientation Angles.

The Stokes parameters are retrieved by multiplying the measurements vector, $\bar{V}$, by the inverse of the above $4 \times 4$ matrix. In this example, that inverse matrix is given by

$$
\left[\begin{array}{cccc}
0 & 1 & 1 & 0 \\
2 & -1 & -1 & 0 \\
-2 & -1-\sqrt{2} & -1+\sqrt{2} & 4 \\
0 & 1 & -1 & 0
\end{array}\right]
$$

In order to accomplish polarimetric imaging with this instrument, an image is taken at each of four angular orientations of the quarter-wave plate. The four signal levels associated with each pixel are then multiplied by the inverse matrix to arrive at the four Stokes parameters for that pixel.

\section{DESIGN OF COMPLETE POLARIMETER}

The complete-polarimeter configuration that we have implemented requires the use of a fixed wire-grid polarizer and a rotating retarder. The typical retardance that is used in this configuration is $90^{\circ}$. Because of the latitude afforded to us by the fabrication of a formbirefringent retarder, we decided to search for the optimal retardance and retarder rotation angles.

The search for optimal retarder rotation angles has been carried out previously. ${ }^{1,2}$

\section{Selection of Retardance and Retarder Orientation Angles}

Data are collected with the complete polarimeter by taking a series of measurements through analyzing elements in various configurations. Assembling the measured values into a vector $\bar{P}$, the operation of an ideal system can be described by a matrix multiplication as

$$
\vec{P}=\mathbf{W} \bar{S}
$$

where $\vec{S}$ is the Stokes vector of the incident beam that is to be determined. W is referred to as the system matrix, and is determined by the Mueller matrices for each configuration of the analyzer and the polarization sensitivity of the detector. In the $k^{\text {th }}$ measurement, the detector receives light characterized by a Stokes vector given by

$$
\mathbf{M}_{k} \vec{S}
$$

where $\mathbf{M}_{\mathrm{k}}$ is the Mueller matrix for that analyzer configuration. Assuming the response of the detector is linearly related to the Stokes vector, the $k^{\text {th }}$ measurement can be described as

$$
P_{k}=\bar{A} \cdot\left(\mathbf{M}_{k} \bar{S}\right)=\left(\vec{A}^{t} \mathbf{M}_{k}\right) \vec{S}
$$


Here $\vec{A}$ is a column vector whose values are essentially detector responsivities for each Stokes vector component. Thus the $k^{\text {th }}$ row of $W$ is given by

$$
\vec{A}^{t} \mathbf{M}_{k}
$$

where the superscripted $t$ represents the transpose operation. In all our numerical work we have assumed a polarization insensitive detector and set

$$
\vec{A}=\left[\begin{array}{l}
1 \\
0 \\
0 \\
0
\end{array}\right] .
$$

As Equation 3 indicates, an estimate $\hat{S}$ of the incident Stokes vector $\vec{S}$ can be reconstructed from the measurements as

$$
\hat{S}=\mathbf{W}^{+} \bar{P}
$$

where $\mathbf{W}^{+}$is the pseudo-inverse of $\mathbf{W}$.

Retardance and retarder orientation angles were chosen by considering $\mathbf{W}$ for a system consisting of an ideal retarder followed by an ideal wire grid polarizer oriented to pass horizontally polarized light, assuming four measurements are taken, each with the retarder at a different angle. A numerical minimization was performed using Mathematica on the $\mathrm{L}_{2}$ condition number of $\mathbf{W}$ as a function of those five variables, i.e., retardance and four retarder angles. The $\mathrm{L}_{2}$ condition number of $\mathbf{W}$ is the ratio of its largest to its smallest singular value. Since singular values are necessarily non-negative, the condition number may take on values of unity or greater. In the reconstruction process given by Equation 8 , singular vector components of the measurement vector $\bar{P}$ are effectively divided by the singular values of W. Amplification of the noise in the measurements occurs if any of the singular values are very small. It should be noted that for a reasonable system design, it will not be the case that all the singular values will be very small, since that would mean no light is able to get through the system and the measurements are all essentially zero. Hence a condition number close to unity, indicating that all the singular values are about the same size, is considered favorable. A large condition number signals at least one small singular value, and is undesirable.

The results of our numerical minimization of the condition number indicate that $135^{\circ}$ retardance is usually more favorable than the typical $90^{\circ}$ wave retardance, regardless of the retarder angles used. The set of retarder angles (of the fast axis relative to horizontal) $\left\{51.7^{\circ}\right.$, $\left.15.2^{\circ},-15.1^{\circ},-51.7^{\circ}\right\}$ with a retardance of $131.8^{\circ}$ was found to yield a favorable condition number of about 1.73. For comparison, a system using a quarter-wave plate with angles of $0^{\circ}, \pm 45^{\circ}$, and $22.5^{\circ}$ would have a condition number of about 5.5 . 


\section{Effect of Non-ideal Retarder}

The form birefringent retarder exhibits diattenuation which would be expected due to the differences in Fresnel reflection losses resulting from the difference in effective index of refraction for the TE and TM modes in the grating. As a consequence, the form-birefringent retarder is not an ideal retarder. Advantageous retarder orientation angles for the actual system, i.e., taking the retarder's diattenuation into account, were obtained by numerical

minimization of the condition number of the system matrix that includes the diattenuation of the retarder. The angles listed in the previous section were used as starting points. This analysis yielded the angles: $21.3^{\circ}, 57.1^{\circ}, 132.6^{\circ}$, and $164.1^{\circ}$.

\section{Tolerance to Variation of Retardance with Wavelength and Angle of Incidence}

In general the retardance of a retarder may vary with parameters such as wavelength and angle of incidence. An analysis was carried out to estimate how much variation could be present without seriously degrading the polarimeter's performance. We suppose that the incident beam which is to be analyzed is composed of a distribution of components each of which undergo a different retardation in the retarder. For example, in a collimated beam with a broad spectral bandwidth, the components could be identified by wavelength. If the retarder were located in a converging or diverging monochromatic beam, angle of incidence on the retarder would serve as the identifier. Describing the distribution of the beam's Stokes vector over retardance $\delta$ effected by the retarder by a density function $\vec{S}_{\delta}$, each component contributes an amount

$$
d \vec{P}=\mathbf{W}(\delta) d \vec{S}=\mathbf{W}(\delta) \bar{S}_{\delta} d \delta
$$

to the measurements recorded by the instrument. Here $\mathbf{W}(\delta)$ is the system matrix for a given retardance angle $\delta$. The vector $\bar{P}$ of measurements can then be expressed as

$$
\vec{P}=\int \mathbf{W}(\delta) \vec{S}_{\delta} d \delta
$$

If the distribution $\bar{S}_{\delta}$ is assumed uniform (i.e. independent of $\delta$ ) over an interval $\left[\delta_{1}, \delta_{2}\right]$ and zero elsewhere, it can be factored out of the integral yielding

where

$$
\bar{P}=\Omega \bar{S}_{\delta}
$$

$$
\Omega=\int_{\delta_{1}}^{\delta_{2}} \mathbf{W}(\delta) d \delta .
$$


Thus the integrated system matrix $\Omega$ plays the same role for the distribution $\vec{S}_{\delta}$ as the system matrix W plays for a single Stokes vector $\vec{S}$, and a study of the conditioning of $\Omega$ will yield information on the tolerable range of retardances.

It should be noted that the assumption of uniform $\vec{S}_{\delta}$ may not be realistic for real sources. This assumption can be considered in two parts: first that the direction of $\bar{S}_{\delta}$ is constant and second that its magnitude is constant in the interval $\left[\delta_{1}, \delta_{2}\right]$ and zero elsewhere. We have been aware since the start of this project that it may be necessary to make the first part of the assumption in order to allow useful interpretation of the data. Assuming $\bar{S}_{\delta}$ to be constant in direction is then not a great concession, but it does mean that this analysis will not provide any insight into how much directional variation in $\bar{S}_{\delta_{0}}$ is tolerable, or how much error such variation would cause if present and not accounted for. Since our goal in this analysis is simply an estimate of tolerances, and not a detailed simulation of the system's performance, approximating the variation of the magnitude $\vec{S}_{\delta}$ with $\delta$ as a rectangular band (as stipulated in the second part of the assumption) is reasonable.

Figure 1 shows the results of the conditioning analysis of $\Omega$ as a function of the interval limits $\delta_{1}$ and $\delta_{2}$. These results assume a polarization analyzer consisting of a rotatable $135^{\circ}$ retarder followed by a linear polarizer, with measurements taken at the favorable retarder orientations obtained in our optimization procedure. The plots indicate that the condition number increases slowly as $\delta_{1}$ and $\delta_{2}$ diverge, allowing a range of angles as great as $\pm 20^{\circ}$ with only $10 \%$ increase in condition number. This is a fairly liberal tolerance, and the peakto-peak variation of retardance with wavelength in the design for the form-birefringent retarder fell well within its limits.
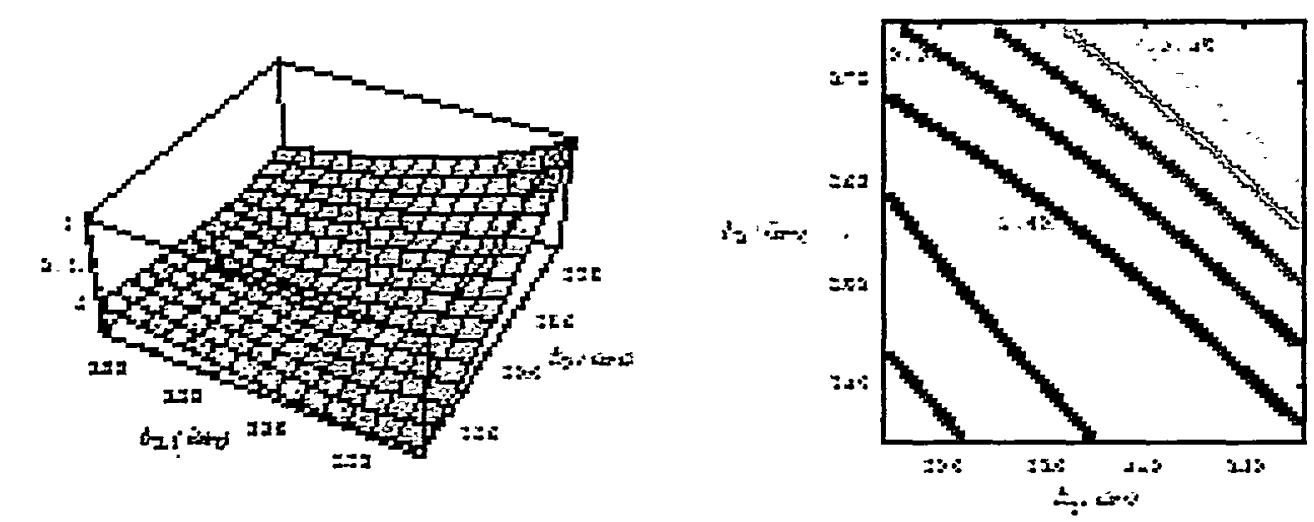

Figure 1. Surface and contour plots showing the variation of condition number for the integrated system matrix as a function of interval limits $\delta_{1}$ and $\delta_{2}$. 


\section{INTERPRETATION OF OPTIMAL COMPLETE POLARIMETER DESIGN USING THE POINCARÉ SPHERE}

The retardance and the retarder-orientation angles can be interpreted in the context of the Poincaré sphere, a construct introduced briefly here and described in more detail in references 3 and 4 . The components of a Stokes vector $\bar{S}$,

$$
\vec{S}=\left[\begin{array}{c}
S_{0} \\
S_{1} \\
S_{2} \\
S_{3}
\end{array}\right]
$$

for completely polarized light (light with no randomly polarized component), satisfy the constraint

$$
S_{0}=\sqrt{S_{1}^{2}+S_{2}^{2}+S_{3}^{2}}
$$

This describes a sphere, named after Poincaré, of radius $S_{0}$ in a coordinate space in which $S_{1}$, $S_{2}$, and $S_{3}$ are plotted on orthogonal axes. The Poincare sphere is a useful construct for visualizing polarization states. Each point on the surface of the sphere represents a different polarization state. The radius $S_{0}$ represents the total irradiance of the beam, while the longitude and latitude represent respectively the orientation and eccentricity of the polarization ellipse (see Figure 2). Appendix A contains instructions for making a paper model of the Poincaré sphere, which is useful for visualization.

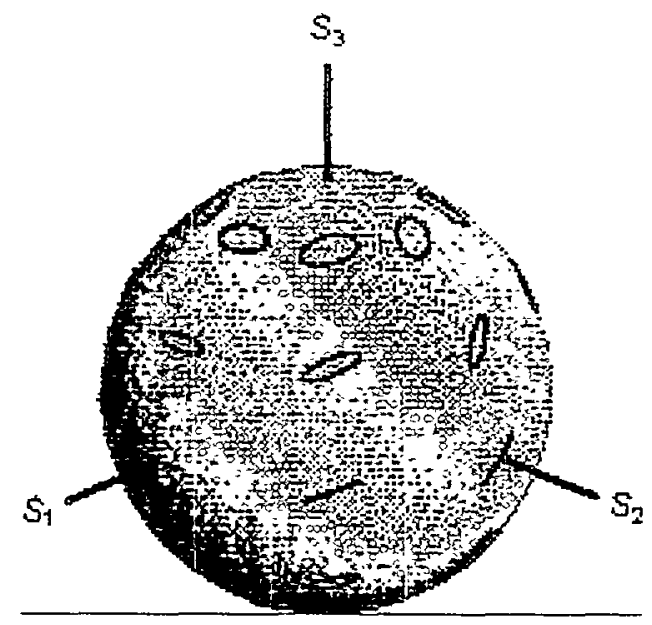

Figure 2. A representation of the Poincaré sphere. The polarization ellipses for several polarization states are shown centered on the corresponding Poincaré sphere locations.

Ambirajan and Look ${ }^{1,2}$ have suggested an interesting interpretation of the question of optimal analyzer configurations which relates to the Poincare sphere. They consider a system with a quarter-wave plate followed by a linear polarizer, and allow both elements to be rotated. This is not equivalent to the system we analyze (in which the waveplate retardance is left as a variable, but the orientation of the polarizer is fixed), but much of their 
mathematics applies to our system. Following Ambirajan and Look's lead, we note that each row of the system matrix $\mathbf{W}$, taken as a vector, is an element of the Stokes vector space. Furthermore, all four of these vectors have the same value of $S_{0}$ and, since they satisfy the condition in Equation 14, represent completely polarized states. Ambirajan and Look point out that when the latter three components of these vectors are plotted on the Poincare sphere, they form the vertices of a tetrahedron, and the volume of the tetrahedron is proportional to the determinant of $\mathbf{W}$.

The determinant is sometimes used in place of the condition numbers as a merit function for optimization. A determinant close to zero indicates that the matrix is nearly singular, while a determinant with large absolute value indicates favorable conditioning. Thus the problem of optimizing the system (with respect to the matrix determinant) can be visualized as a matter of moving the tetrahedron vertices on the Poincare sphere to maximize the tetrahedron's volume. Geometry shows that the volume of the tetrahedron is maximized when it is equal-sided. Hence it is desirable to space the tetrahedron vertices as far apart as possible on the Poincaré sphere. In fact, as illustrated in Figure 3, the configuration we obtain by minimizing the $\mathrm{L}_{2}$ condition number exhibits this property without requiring rotation of the polarizer. We note that the configuration we have found leads to favorable conditioning with respect to both the $\mathrm{L}_{2}$ condition number and determinant criteria.

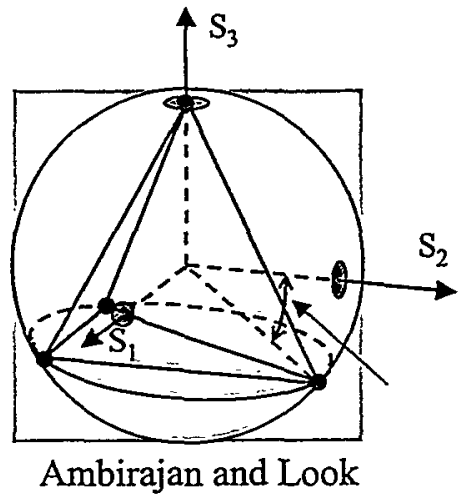

(a)

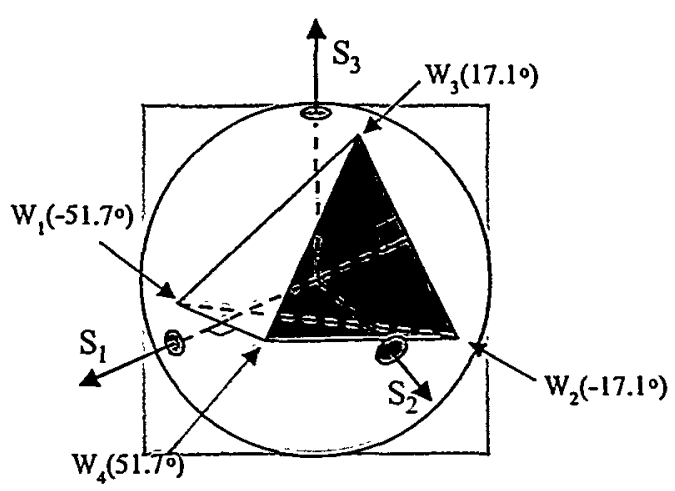

(b)

Figure 3. Part a shows the tetrahedron for a configuration reported by Ambirajan and Look. Our configuration, illustrated in part $b$, similarly describes an equal-sided tetrahedron.

The interpretation of the optimization process on the Poincare sphere offers the hope of an easily visualized and intuitive interpretation of the performance of a polarimeter, which would be helpful in guiding the design and optimization of future systems. It has, however, a number of aspects that have yet to be spelled out. For example, the significance of the polarization states corresponding to the location of the tetrahedron vertices on the Poincare sphere has not been addressed. It should also be noted that the foregoing discussion assumed a perfect retarder. When the effects of the diattenuation observed in the form-birefringent retarder fabricated for our instrument are included in the description of the system, the rows 
of the system matrix $\mathbb{W}$ take on a partially polarized component. Investigation of such issues is left for further work.

\section{DESIGN AND FABRICATION OF THIE RETARDER PLATE}

On the basis of the condition number minimization and tolerance studies, a value of $133^{\circ}$ was chosen as the target for the retarder, with a tolerance of $\pm 20^{\circ}$. A subwavelength component design was chosen to meet this specification for the waveplate across the broad application waveband of 4.42-5 $\mu \mathrm{m}$. This subwavelength element utilizes form birefringence to introduce an effective refractive index difference, and thus a phase delay difference, between the orthogonal polarization components of a normally incident plane wave. The subwavelength element is a simple rectangular profile grating on GaAs. Grating depth determines the retardance angle introduced as the light is transmitted through the element. Grating pitch and duty cycle determine the useful waveband of the component. Initially, effective medium theory was used to identify the parameter space as this is a relatively quick way to approximate the retardance angle and waveband of the element. A rigorous coupled wave analysis code (KAPPA, Lifeng Li) was used to more accurately simulate the grating's effect upon an incident plane wave.

The final design simulation is shown below in Figure 4 for wavelengths from 3 to 6 $\mu \mathrm{m}$. The retarder's performance is shown relative to $\pm 10^{\circ}$ tolerance bars indicated by the horizontal rectangle. The grating pitch is 1.17 microns, the duty cycle is 0.56 , and the grating depth is 1.78 microns.

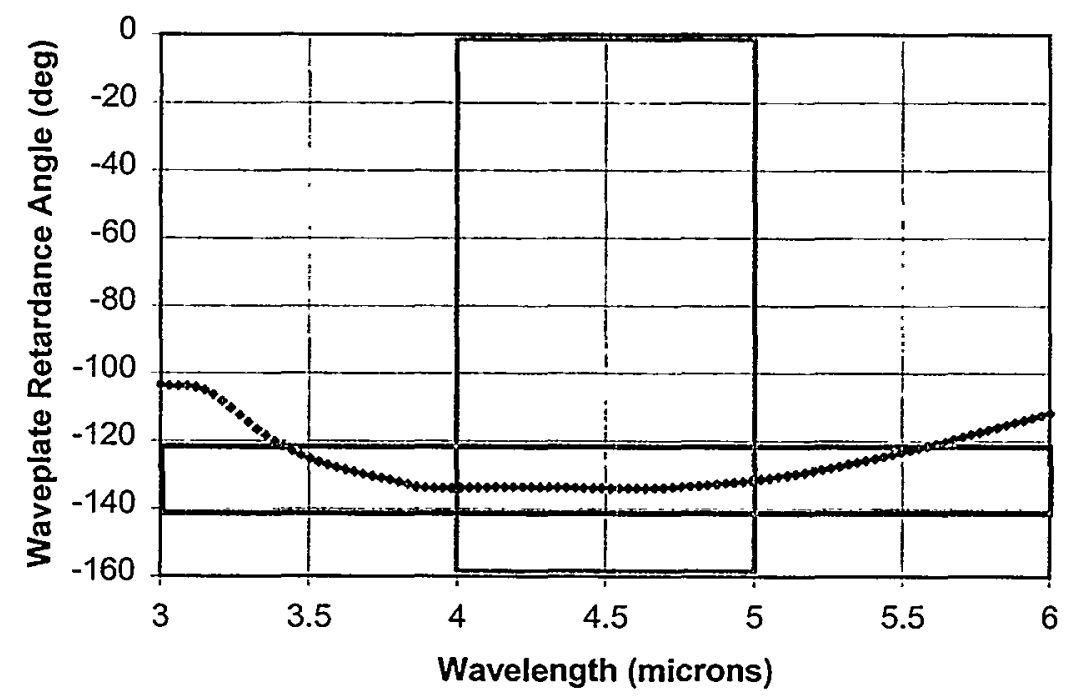

Figure 4. Simulated waveplate retardance angle as a function of incident light wavelength for the retarder as it was designed. The vertical-rectangle indicates the spectral range for which the retarder is intended.

A $20 \mathrm{~mm}$ by $20 \mathrm{~mm}$ waveplate mask was defined directly on the GaAs with an ebeam process. Writing the mask with the e-beam took a significant amount of time 
(approximately 15 hours write time), but the time was necessary to obtain the small feature sizes and tight control upon the grating duty cycle. Reactive Ion Beam Etch (RIBE) was used to etch the grating to the correct depth and obtain smooth and vertical side walls for the rectangular profile grating. A Scanning Electron Microscope (SEM) picture of the fabricated grating is shown in Figure 5.

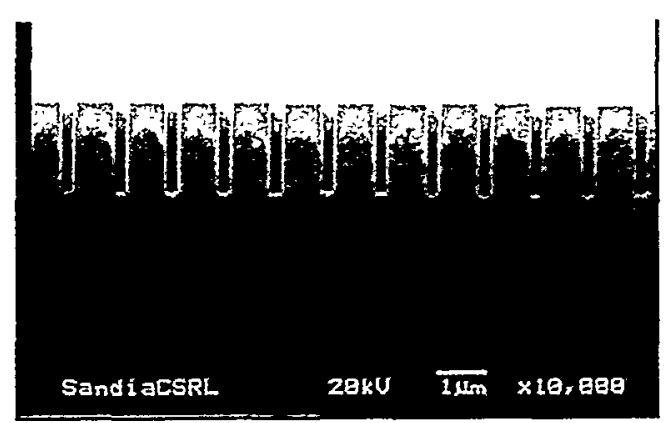

Figure 5. SEM picture of the rectangular profile grating as fabricated.

A simulation of the waveplate's performance as fabricated was performed using the grating parameters measured in the SEM. Here the grating pitch was 1.12 microns, the duty cycle was 0.64 , and the grating depth is 1.83 microns. These results appear in Figure 6, and remain within the desired $133^{\circ} \pm 20^{\circ}$ specification over the $4-5 \mu \mathrm{m}$ waveband. The 4-5 $\mu \mathrm{m}$ waveband is indicated by the vertical rectangle in Figure 6 .

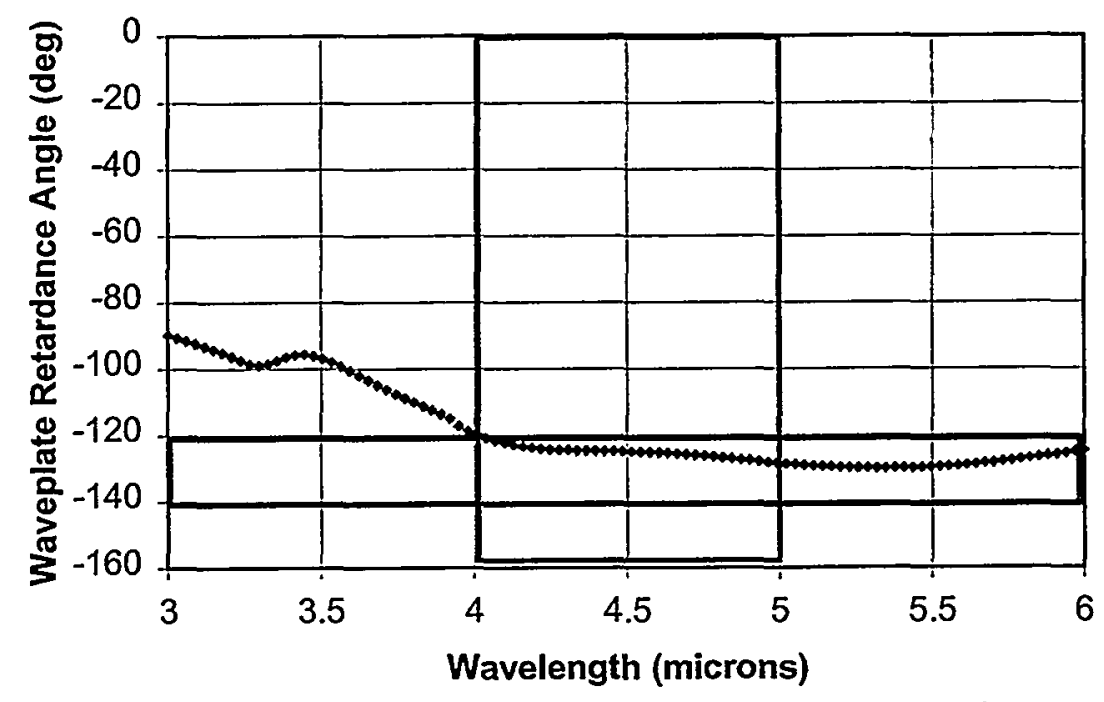

Figure 6. Simulated waveplate retardance angle as a function of incident light wavelength for the component as it was fabricated. 
It is important to note that the retarder is used in transmission. Therefore, the unpatterned surface needs to be polished as any other optical surface in the polarimeter optical train.

\section{CHARACTERIZATION OF POLARIMETER COMPONENTS}

The reconstruction of Stokes parameters from direct measurements requires accurate knowledge of the polarizing elements that comprise the complete polarimeter, i.e., the wiregrid polarizer and the retarder plate. Several systems were used to characterize polarization elements. We began by constructing an experimental set-up (see Figure 7) capitalizing on the linearly polarized output of a $3.39 \mu \mathrm{m}$ HeNe laser (Jodon HeNe $40 \mathrm{~mW}$ laser, Model HN20G-IR3.39). Note that this wavelength is outside the system's band. The measurements are nonetheless useful for characterization of wire grid polarizers, since their extinction ratio tends to improve with increasing wavelength (as the grid period is pushed further into the sub-wavelength regime). The performance of the retarder at $3.39 \mu \mathrm{m}$ is also of interest for comparison with simulations (Figure 4 and Figure 6) which included that wavelength. An additional $632.8-\mathrm{nm} \mathrm{HeNe}$ laser and an adjustable iris were used to align the optical train of the characterization set-up.

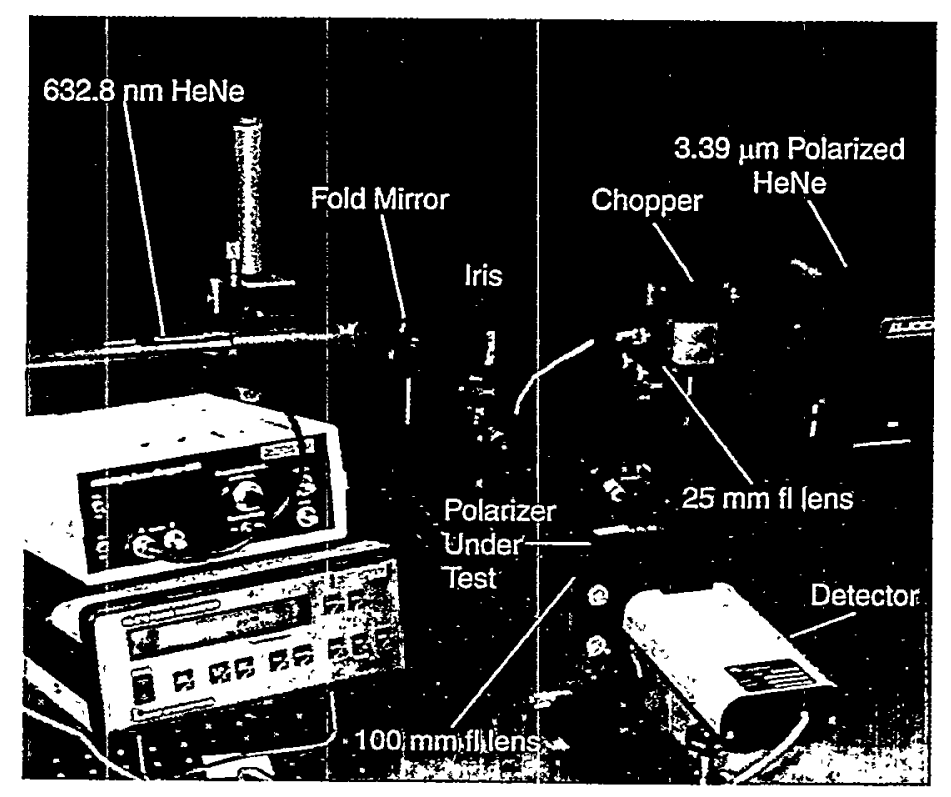

Figure 7. Polarizer characterization set-up. See text for details.

Light emitted by the HeNe laser was first chopped at $28.5 \mathrm{~Hz}$ (HMS Light Beam Chopper 220). Next, light was focused by a $25-\mathrm{mm}$ focal length germanium singlet lens. A fold mirror directed the laser's radiation at the polarizer under test. The distance from the focal plane of the germanium singlet to the polarizer under test was chosen so as to overfill the aperture of the polarizer. The polarizer was followed by a second germanium singlet $(f=100$ $\mathrm{mm}$ ) that focused the light transmitted through the polarizer onto a pyroelectric detector (Laser Precision, Inc. RKP-575, RKP-5710). 
The output of the radiometer was recorded via a GPIB bus using a personal computer and LabVIEW software (National Instruments, version 4.1). The virtual-instrument (VI) interface is shown in Figure 8 and the corresponding VI program is provided in Appendix B.

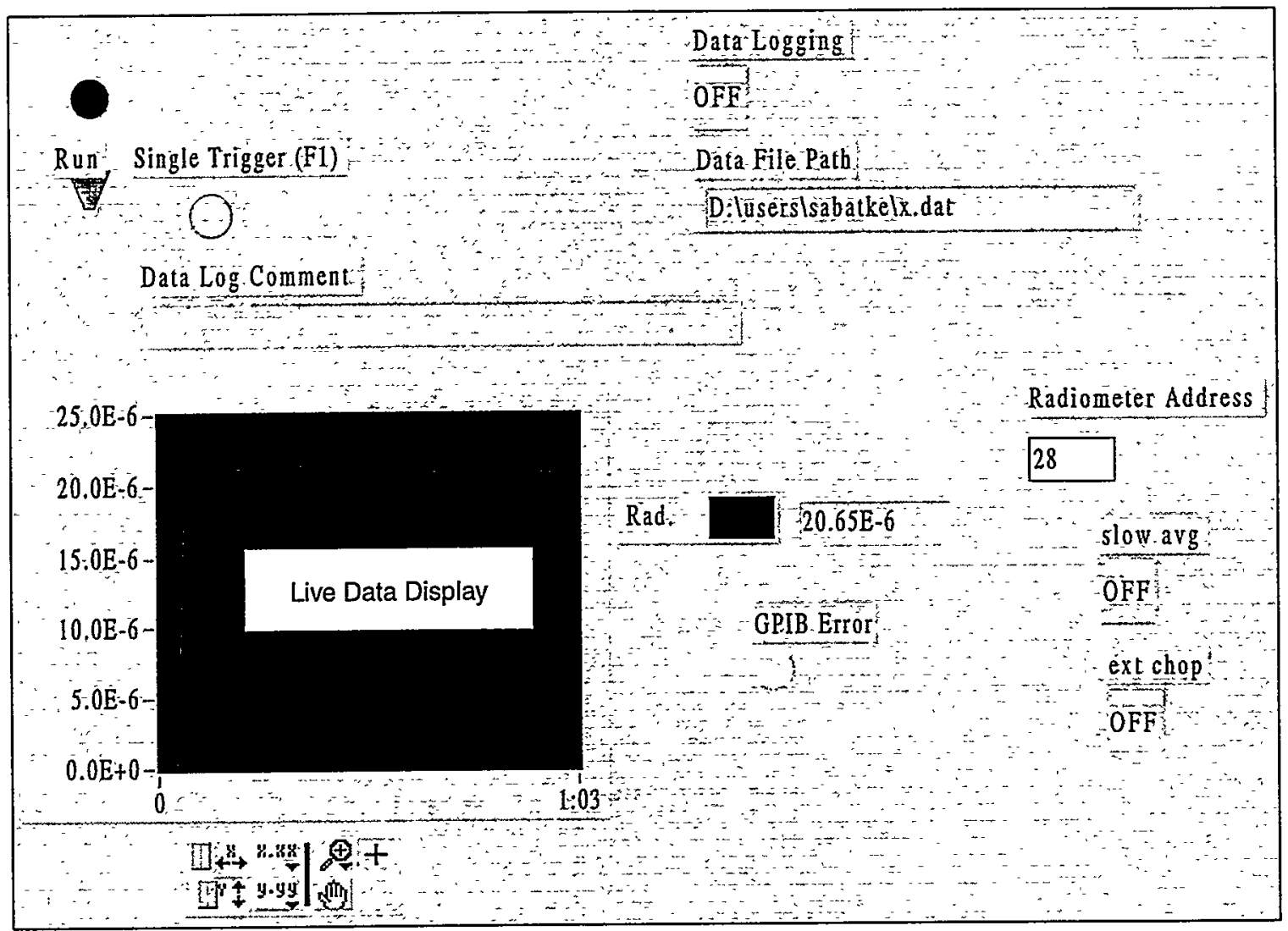

Figure 8. Virtual instrument interface created to control the characterization set-up.

\section{Characterization Results}

Wire Grid Polarizer Characterization

The $3.39 \mu \mathrm{m}$ characterization set-up was used to measure the extinction ratios of the 1" diameter wire grid polarizers obtained from Optometrics. The maximum and minimum of the transmitted power were recorder as the polarizer was rotated, and the extinction ratio was calculated as the ratio of these power measurements. Extinction ratios of $47.5 \pm 6.7$ and 142 \pm 25 were obtained for the $\mathrm{CaF}_{2}$ and $\mathrm{ZnSe}$ wire-grid polarizers, respectively.

\section{Form-birefringent Retarder Characterization}

The form-birefringent retarder element was characterized in table-top systems at 3.39 $\mu \mathrm{m}$ and over a broad band (4.5-5.5 $\mu \mathrm{m})$. In addition, another form-birefringent retarder from a second fabrication run was characterized at multiple wavelengths using a Fourier transform infrared spectrometer (FTIR). The basic method of all three measurements was the same. Polarized light, obtained from a polarized source (in the case of the $3.39 \mu \mathrm{m} \mathrm{HeNe}$ ) or with the use of a wire grid polarizer, was transmitted through the waveplate at a specific rotation 
angle, then through a fixed angle infrared polarizer, and to a detector. The power registered by the detector was measured as a function of waveplate orientation angle. Mueller matrices can be used to model this measurement process with the retardance $\delta$ of the waveplate left as a free parameter. Each optical component in the experiment has a corresponding Mueller matrix. The rotation matrix for an angle $\theta$ is defined as

$$
R[\theta]=\left[\begin{array}{cccc}
1 & 0 & 0 & 0 \\
0 & \cos (2 \theta) & -\sin (2 \theta) & 0 \\
0 & \sin (2 \theta) & \cos (2 \theta) & 0 \\
0 & 0 & 0 & 1
\end{array}\right]
$$

A linear diattenuator transmits linear polarization along one axis with intensity transmittance $q$, and along the orthogonal axis with intensity transmittance $r$. A non-ideal polarizer with an extinction ratio $\varepsilon$ can be modeled with this expression where $q=1$ and $r=$ $1 / \varepsilon$. Its Mueller matrix when its $q$-axis is rotated at an angle $\theta$ is given by

$$
L D[\theta, q, r]=\frac{1}{2} R[\theta] \cdot\left[\begin{array}{cccc}
q+r & q-r & 0 & 0 \\
q-r & q+r & 0 & 0 \\
0 & 0 & 2 \sqrt{q r} & 0 \\
0 & 0 & 0 & 2 \sqrt{q r}
\end{array}\right] \cdot R[-\theta] .
$$

Finally, a linear retarder with fast axis at angle $\theta$ and retardance $\delta$ has the Mueller matrix

$$
L R[\theta, \delta]=\frac{1}{2} R[\theta] \cdot\left[\begin{array}{cccc}
1 & 0 & 0 & 0 \\
0 & 1 & 0 & 0 \\
0 & 0 & \cos (\delta) & \sin (\delta) \\
0 & 0 & -\sin (\delta) & \cos (\delta)
\end{array}\right] \cdot R[-\theta]
$$

With these expressions, the experiment was modeled with the matrix equation

$$
\text { signal }=a\left[\begin{array}{llll}
1 & 0 & 0 & 0
\end{array}\right] \cdot L D\left[0,1, \frac{1}{\varepsilon}\right] \cdot L R[\theta, \delta] \cdot L D[\theta, q, r] \cdot\left[\begin{array}{l}
1 \\
1 \\
0 \\
0
\end{array}\right] .
$$

The right-most parts of this expression correspond to elements encountered first in the optical train. The vector $[1,1,0,0]$ on the right is the Stokes vector for the incoming (horizontally) polarized light. The waveplate is modeled as a combination linear diattenuator and linear retarder. This provides sufficient generality to account for the angular dependence of the (Fresnel) intensity transmission due to the variation of effective index as a function of 
rotation angle. The last matrix represents another polarizer, and the last vector $[1,0,0,0]$ describes the detector's response which is sensitive only to the $S_{0}$ Stokes parameter. (Note that Equation 18 is the equivalent of Equation 5 for the particular configuration of polarization elements involved in the test setup.) The expression for signal as a function of $\theta$ can be used in a least-squares fit of the data to estimate the retardance $\delta$ as well as the extinction ratio of the element's diattenuation.

Technically, this measurement technique allows determination of $\cos \delta$ but not $\sin \delta$, so that $\delta$ is not determined uniquely. This amounts to an ambiguity in the sign of $\delta$, or equivalently an inability to distinguish between the fast and slow axes. We know on theoretical grounds how the retarder axes are situated relative to the grating lines, so we can circumvent the retardance sign ambiguity. The fast axis is normal to the grooves of the grating. A visible laser beam provides a practical means of determining the direction of the fast axis. When the beam is reflected from the sample at normal incidence, the diffracted orders are dispersed along a direction normal to the grooves and therefore parallel to the fast axis.

\section{Retarder Characterization at $3.39 \mu \mathrm{m}$}

The tabletop system described earlier and shown in Figure 7 was used to characterize the retarder at a wavelength of $3.39 \mu \mathrm{m}$. Several power readings were taken at each angular setting in order to generate an estimate of the error (in the mean) for each data point. The data are shown as points in the plot below. The error bars are considerably smaller than the point diameters. A careful measurement of overall transmittance was not carried out, but it was estimated to be approximately $35 \%$. Figure 9 shows the data and fit. The laser was subject to slow drifts (of a $1 / f$ nature) so that, despite the inclusion of a linear term in the amplitude of the fitting function in an attempt to compensate, the fit quality is poor. Values of $90.4^{\circ}$ and 1.13 were obtained for retardance and extinction ratio respectively. This retardance is in good agreement with the simulated value of about $95^{\circ}$ shown in Figure 6.

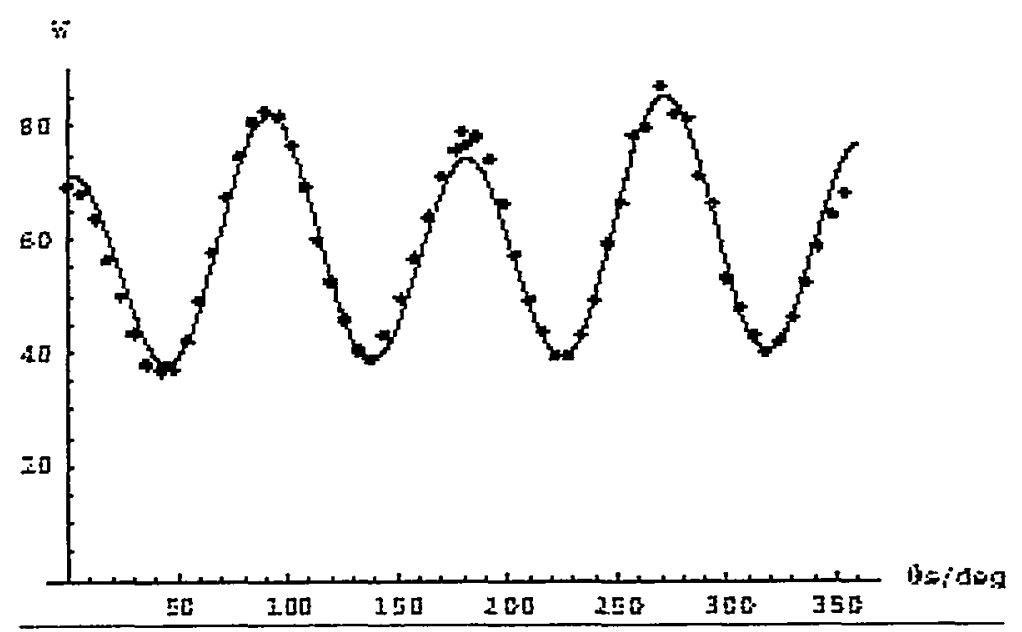

Figure 9. The experimental data taken at $3.39 \mu \mathrm{m}$ and their least-squares fit. 


\subsection{2-5.46 um Broadband Characterization}

In order to circumvent the drifts of the laser's output and to obtain data at the system's operating wavelengths, a similar measurement was carried out using a $1000{ }^{\circ} \mathrm{C}$ blackbody as a source. Radiation from the approximately $1 \mathrm{~mm}$ diameter aperture was chopped (at $28.5 \mathrm{~Hz}$ ), collimated, and passed through a 4.42-5.46 $\mu \mathrm{m}$ bandpass filter (OCLI, W0944-8) and a wire grid polarizer before being sent on to the retarder and second polarizer (Figure 10). A lens after the polarization elements focussed the beam into the detector aperture. Figure 11 shows the data and the fit used to characterize the form-birefringent retarder.

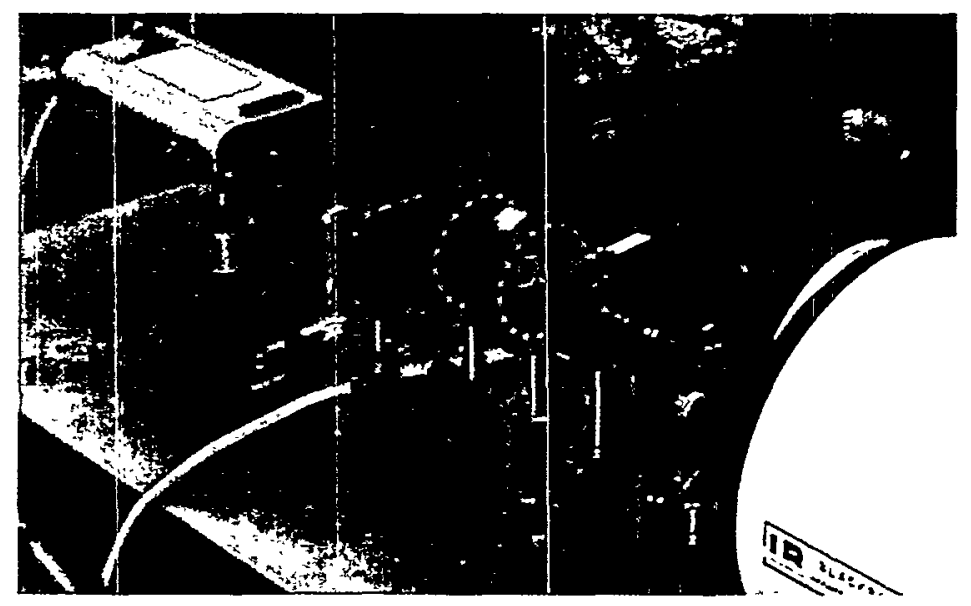

Figure 10. Experimental set-up for at-wavelength, broadband characterization of polarizing elements.

An extra sinusoidal factor was added to the fitting function to account for slight differences between values measured at angles differing by $180^{\circ}$. The effect may be due to decentering of apertures in the system in conjunction with non-uniformities in the properties of polarization elements across their faces. In the approximation that the retarder's polarization properties are uniform across its face, the effect cannot be a due to a polarization property of the retarder since Mueller matrices are invariant under a rotation of $180^{\circ}$. In any event, the extra factor in the fitting function has negligible impact on the retardance and extinction ratio values obtained, but improves the quality of fit (yielding a reduced $\chi^{2}$ value close to unity). Values of $116.8^{\circ} \pm 0.2^{\circ}$ and $1.498 \pm 0.004$ were obtained for the retardance and extinction ratio of the retarder, respectively. These are the values used to compute the system matrix W in the Stokes vector measurements reported later in this report. 


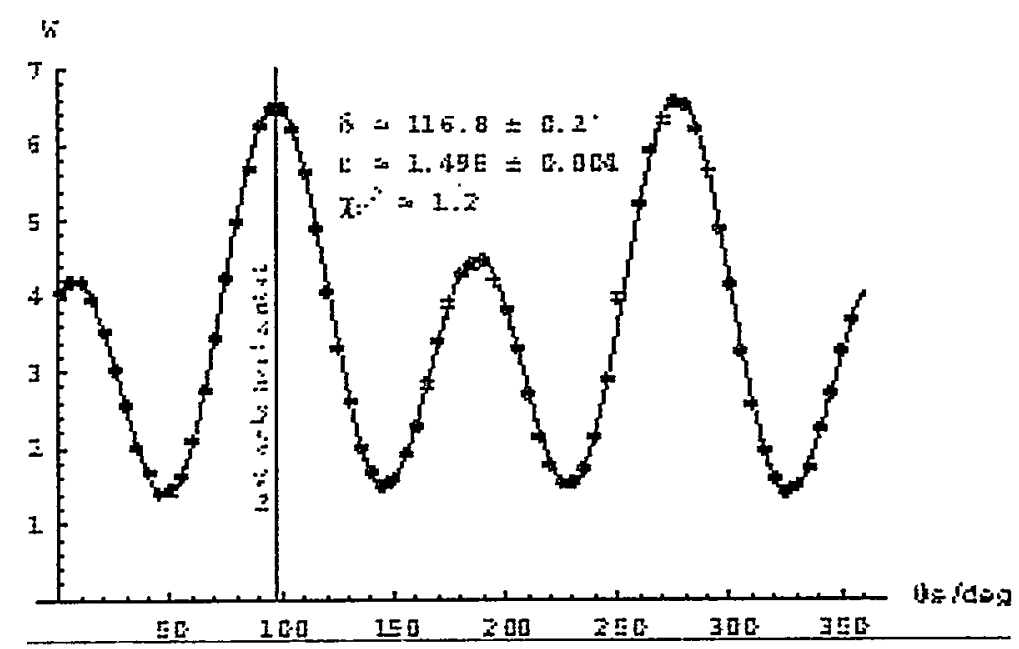

Figure 11. The retarder-characterization data and fit used to determine $\delta$ and $\varepsilon$.

\section{Characterization in an FTIR}

Another retarder from a different fabrication run was characterized by employing the same approach in an FTIR. A gray-body source and infrared polarizer were used to provide the polarized input. The FTIR measures the spectrum of the light transmitted through the system. By carrying out fits to its data at each wavelength, retardance was determined as a function of wavelength. Figure 12 shows the data and fit for a wavelength of $4.5 \mu \mathrm{m}$. Figure 13 shows the measured retardances as a function of wavelength, together with the values obtained from the simulation of the as-fabricated waveplate. Both simulation and measurements are within the target waveplate retardance angle of $133^{\circ} \pm 20^{\circ}$ over the $4-5 \mu \mathrm{m}$ waveband. 


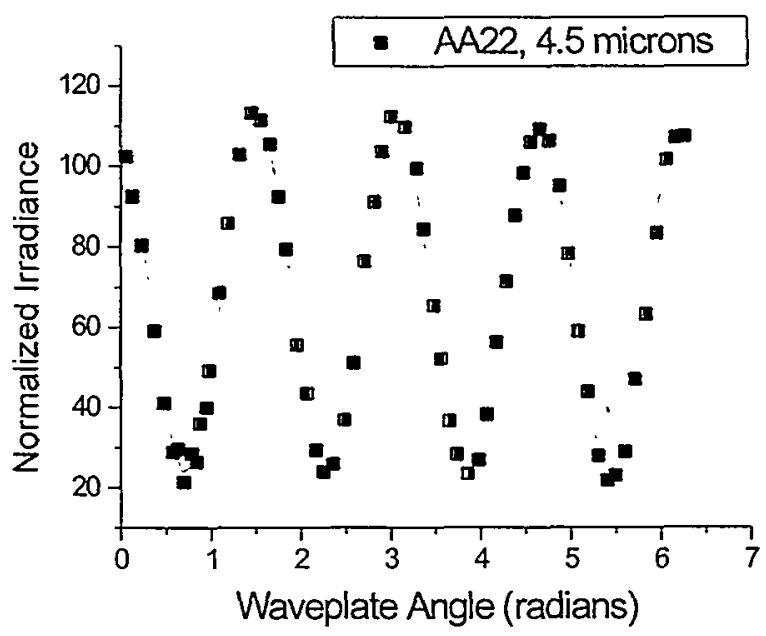

Figure 12. Detected intensity (squares) as a function of waveplate rotation angle for an incident wavelength of 4.5 microns. Curve fit (line) for waveplate retardance angle of $128^{\circ}$.

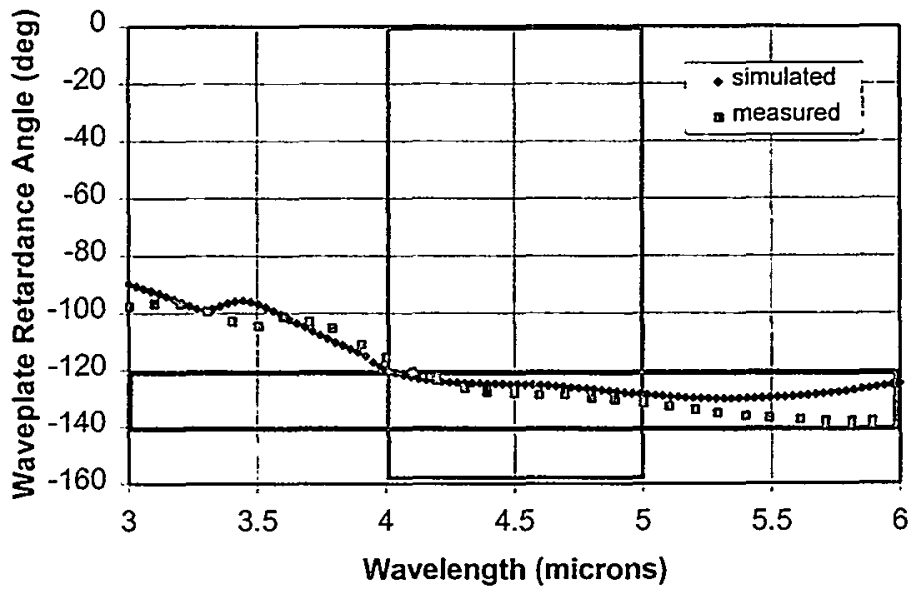

Figure 13. Measured waveplate retardances (squares) and simulated as-fabricated waveplate retardances (diamonds) as a function of incident wavelength.

\section{COMPLETE IMAGING POLARIMETER}

We constructed a complete imaging polarimeter using a PtSi midwave infrared (MWIR) camera, a wire-grid polarizer, and a form-birefringent retarder plate. The wire-grid polarizer was a commercially available optical element (The Optometrics Group, Catalog Nos: 52825 and 5-2625). 


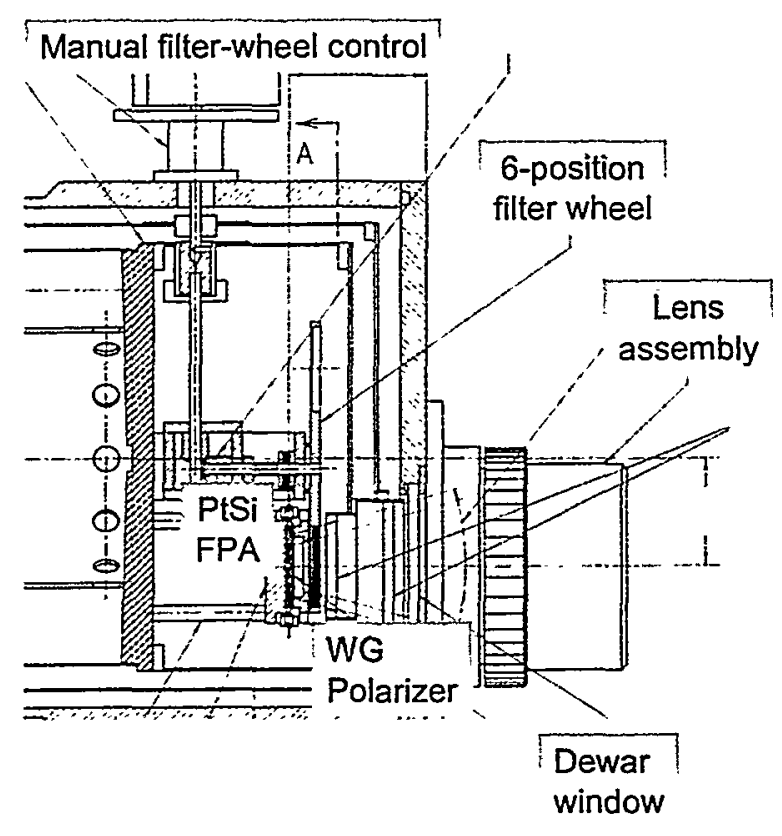

Figure 14. PtSi MWIR camera and location of wire-grid polarizer.

Figure 14 shows the locations of the wire-grid polarizer and the PtSi focal plane array (FPA). The WGP was mounted in a rotating filter wheel and is the last optical element prior to the FPA. The wire-grid polarizer is preceded by a band-pass filter and $\mathrm{a} \mathrm{CaF}_{2}$ window in the dewar. The dewar is of the pour-filled type and its contents are maintained at the temperature of liquid nitrogen, i.e., $77 \mathrm{~K}$.

\section{Dewar Window Considerations}

The original dewar window was made of sapphire $\left(\mathrm{Al}_{2} \mathrm{O}_{3}\right)$ which is birefringent. The orientation of the sapphire window and the crystalline axes determine the effect of the window on incident polarized radiation. The ordinary and extraordinary indices of refraction exhibit the following dependence on wavelength, as expressed through Sellmeier formulae:

$$
\begin{aligned}
& n_{o}^{2}(\lambda)-1=\frac{1.4313493 \lambda^{2}}{\lambda^{2}-(0.0726631)^{2}}+\frac{0.65054713 \lambda^{2}}{\lambda^{2}-(0.1193242)^{2}}+\frac{5.3414021 \lambda^{2}}{\lambda^{2}-(18.028251)^{2}} \\
& n_{e}^{2}(\lambda)-1=\frac{1.5039759 \lambda^{2}}{\lambda^{2}-(0.0740288)^{2}}+\frac{0.55069141 \lambda^{2}}{\lambda^{2}-(0.1216529)^{2}}+\frac{6.5927379 \lambda^{2}}{\lambda^{2}-(20.072248)^{2}} .5
\end{aligned}
$$

Equations 19 and 20 are valid from 0.2 to 5.5 microns and therefore include the MWIR range in which our instrument was designed to operate. Using our 3.39 micron characterization set-up, we estimate the retardance of our sapphire dewar window to be $90^{\circ}$. Birefringence of this magnitude, if not accurately characterized (at wavelength) and accounted for in the system matrix, would have corrupted the data. Since the window was about $3 \mathrm{~mm}$ thick, it constituted a multiple-order waveplate and would have exhibited a strong wavelength dependence in its retardance. Since our instrument is intended to operate over the fairly 
broad band of $4.5-5.0 \mu \mathrm{m}$, such dispersion is not tolerable and the sapphire window was replaced with one of $\mathrm{CaF}_{2}$ (a non-birefringent material).

\section{EXPERIMENTAL RESULTS}

Data was acquired by taking images of the target scene with the retarder oriented to each chosen angle. Pedestal images were also taken for each angle with system's aperture blocked by a liquid nitrogen-cooled sponge. The pedestal images represent the contribution to the signal in the first set of images from thermal emission in the optical system. The pedestal images were subtracted from the corresponding scene images, yielding a set of values for each detector pixel that corresponds to the measurement vector $\vec{P}$. A system matrix W was generated on the basis of our characterization of the form birefringent retarder, the wire grid polarizer, and their orientations. The scene's Stokes vectors were then estimated with the pseudo-inverse of $\mathbf{W}$ as given in Equation 8. No differences were observed between the pedestal images for different retarder orientations, so in practice a single pedestal image could have been used so long as the camera's offset setting was constant and environmental conditions were stable.

\section{Outdoor Experiments: Idling Automobile}

We conducted two experiments that involved man-made objects. The first experiment involved an idling automobile (1994 Volkswagen Jetta). Imaging-polarimetry results from this experiment are shown in the following Figures. The instrument's line of sight made an angle of about $75^{\circ}$ with the normal to the hood of the car.

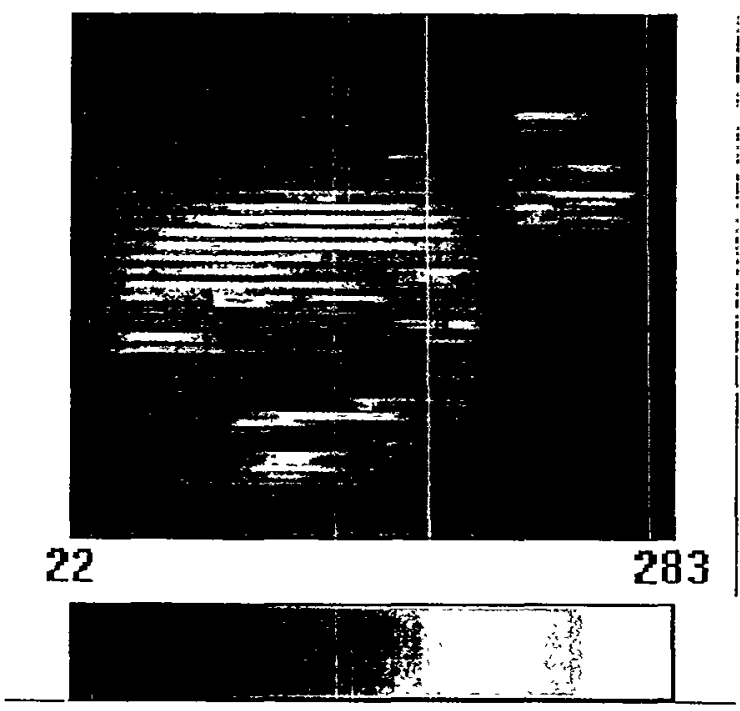

(c) $S_{2}$

(a) $S_{0}$

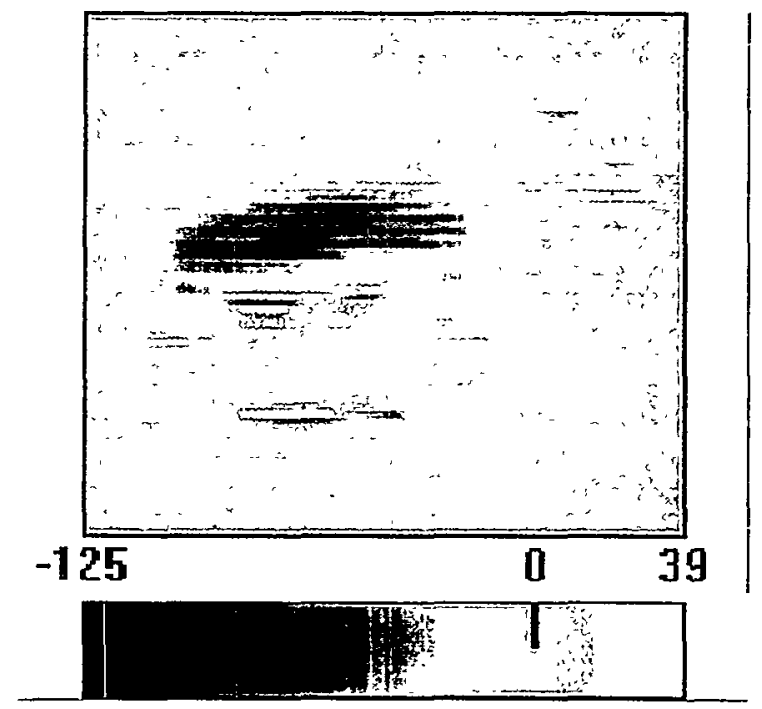

(d) $S_{3}$

(b) $S_{1}$ 

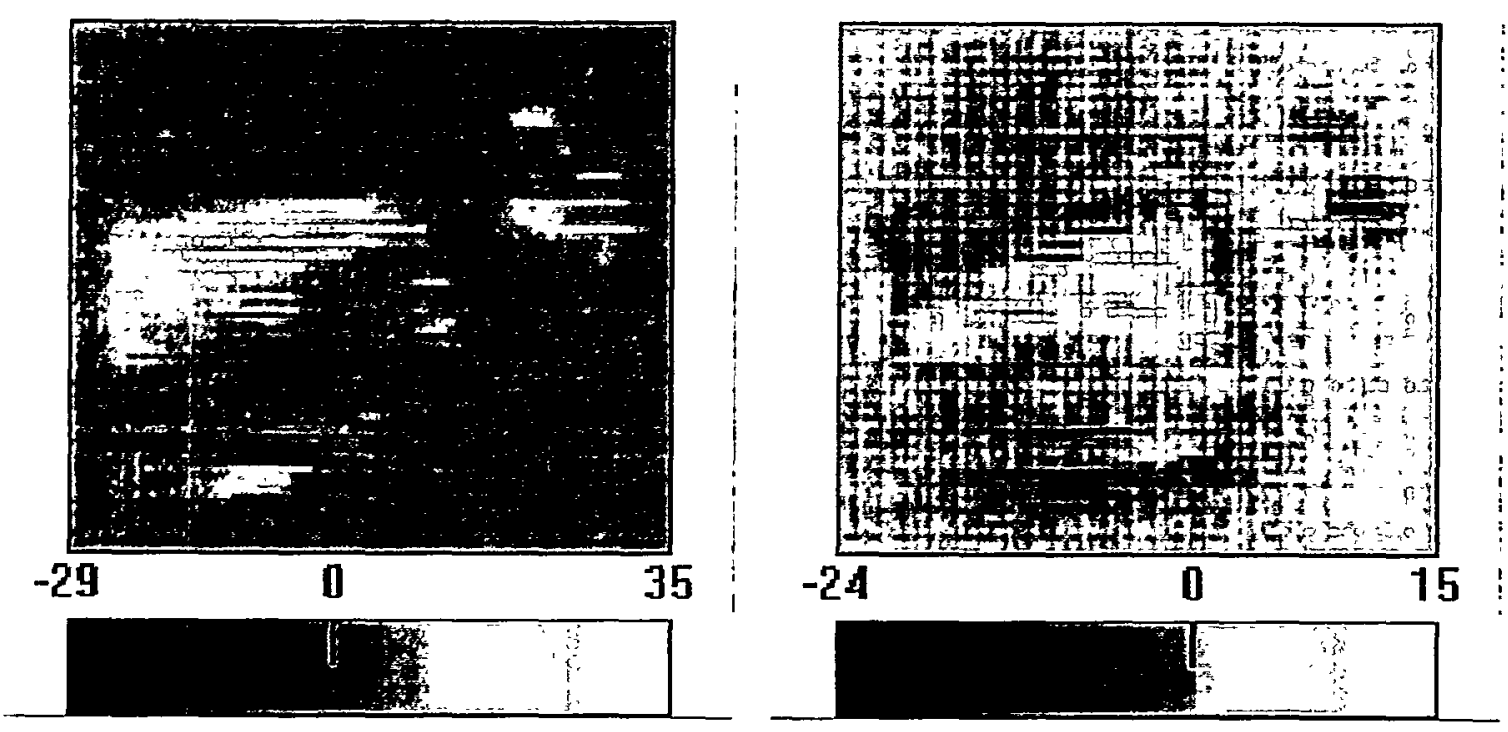

Figure 15. Stokes images of an automobile hood with engine idling. The artifact in the lower right comer of the windshield (indicated in Figure 16) is due to defective pixels in the camera's FPA.

A more quantitative representation of the automobile images is given in Figure 17. The four curves in Figure 17, labeled $S_{0}$ to $S_{3}$, correspond to the same row in the automobile image (see Figure 16). Among the Stokes parameters that express a polarization preference, the dominant signal is due to $S_{1}$, i.e., the horizontal vs. vertical polarization preference. The fact that the sign of $S_{1}$ is negative indicates a preference for vertical polarization. Vertical polarization dominates in the case of emitted radiation. This can be demonstrated by considering Fresnel formulas for transmissivity of a medium-air interface. ${ }^{6}$

The non-zero $S_{2}$ parameter value is most likely caused by a tilt of the car's hood. The magnitude of the $S_{3}$ parameter is comparable to the noise fluctuations notable on the curve labeled $S_{3}$.

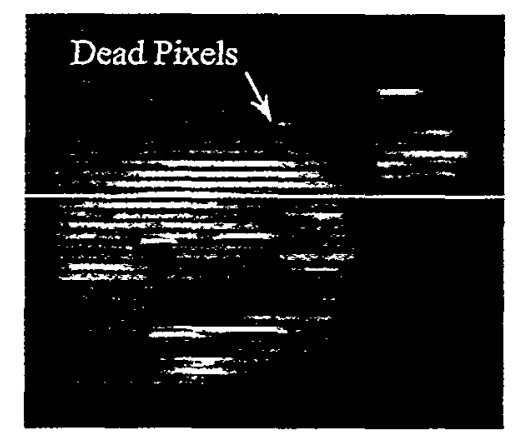

Figure 16. Location of profile taken through automobile image as shown in Figure 15. The indicated dark image segment is due to a defective FPA region. 


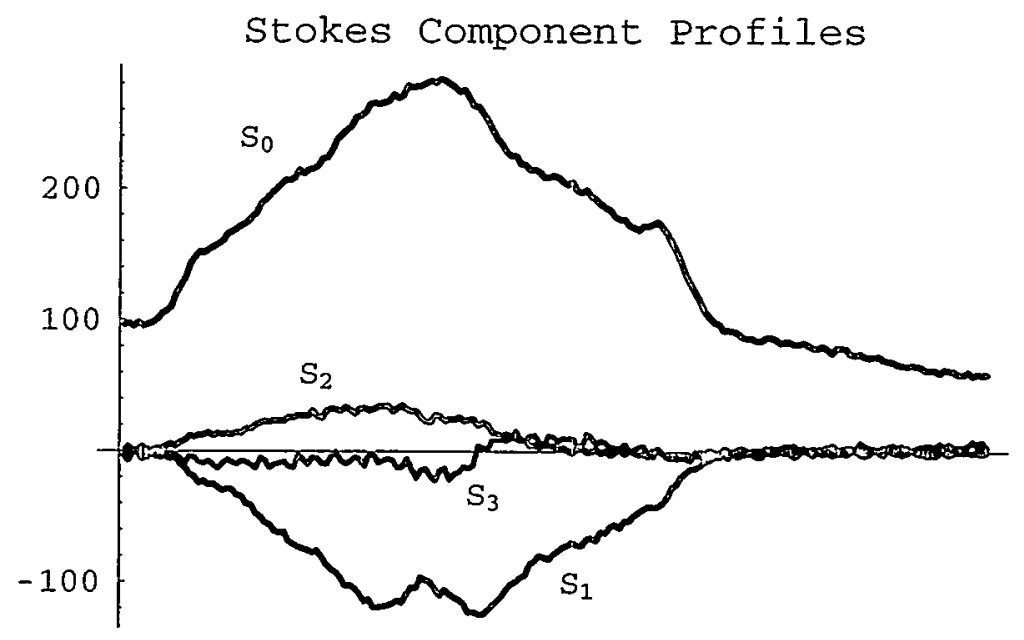

Figure 17. Profiles through automobile image. Each curve corresponds to one of the four Stokes parameters.

The degree of polarization (DOP) and degree of linear polarization (DOLP) are summary measures which are sometimes used to characterize polarization states. These are defined in terms of Stokes vector components as

$$
\begin{gathered}
D O P=\frac{\sqrt{S_{1}{ }^{2}+S_{2}{ }^{2}+S_{3}{ }^{2}}}{S_{0}} \\
D O L P=\frac{\sqrt{S_{1}^{2}+S_{2}^{2}}}{S_{0}}
\end{gathered}
$$

Note that satisfying Equation 14 is equivalent to having a DOP of $100 \%$. The DOP for the chosen profile through the automobile data is plotted in Figure 18, showing a maximum of about $49 \%$. Because the $S_{3}$ component is negligible in this data, the DOP and DOLP are essentially equal. 


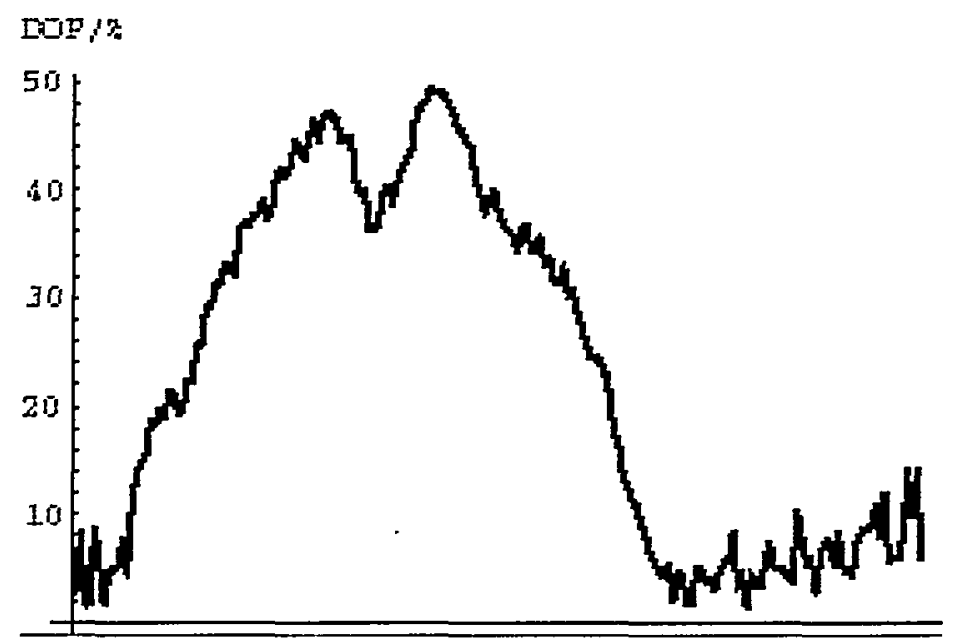

Figure 18. The $\mathrm{DOP}$ (in percent) for the profile of the automobile image in Figure 16. The horizontal axis represents pixels along the profile in the automobile image.

\section{Aircraft}

Imaging-polarimetry data were obtained from a Cessna aircraft at the FICO airstrip in Sahuarita, AZ, under clear-sky conditions. The aircraft was located in direct sunlight on the tarmac and had been heated by the sun. Its engine was not running, however. During the data processing, the raw images were scaled independently in order to correct for drifts in the camera's gain. The scaling factors were determined by constraining a section of the background to reconstruct as random polarization. The background image section was selected based on the expectation of being unpolarized. The operation introduced no more than $5 \%$ change in any of the raw images for this data. Images corresponding to each of the four Stokes parameters are shown in Figure 19.

A strong vertical polarization component from the airplane's windshield is apparent [see Figure 19(b)]. The vertical polarization component is associated with emitted thermal radiation.

It is also interesting to note that the $S_{2}$ parameter's value changes from positive to negative across the windshield. This behavior is consistent with the expected variation in polarization across the windshield due to the windshield's curvature around the front of the aircraft. The effect is small but can be seen distinctly above the noise in the profiles of Figure 20(b).

Figure 21 shows the DOP for the profile, with a maximum of about $16 \%$. 


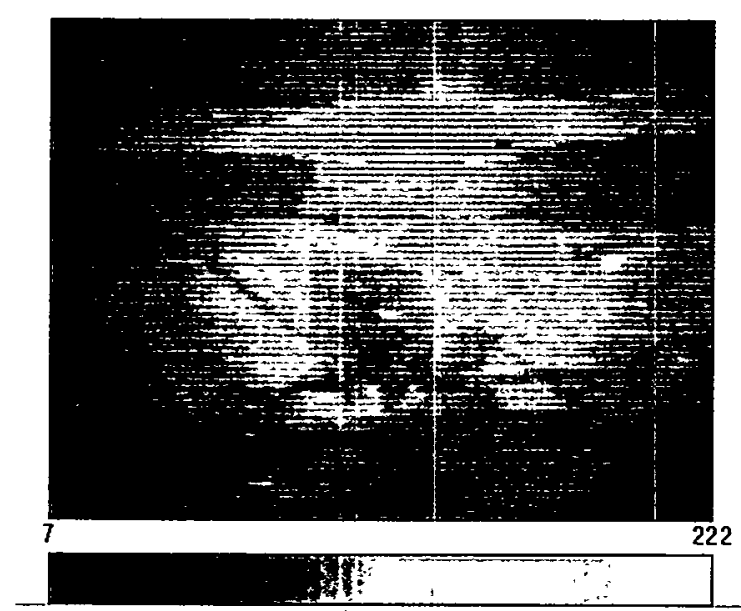

(a) $S_{0}$

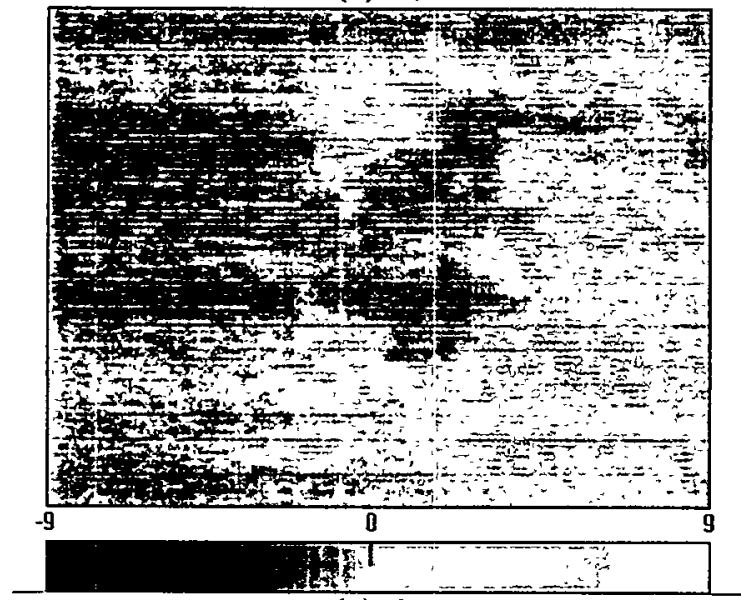

(c) $S_{2}$

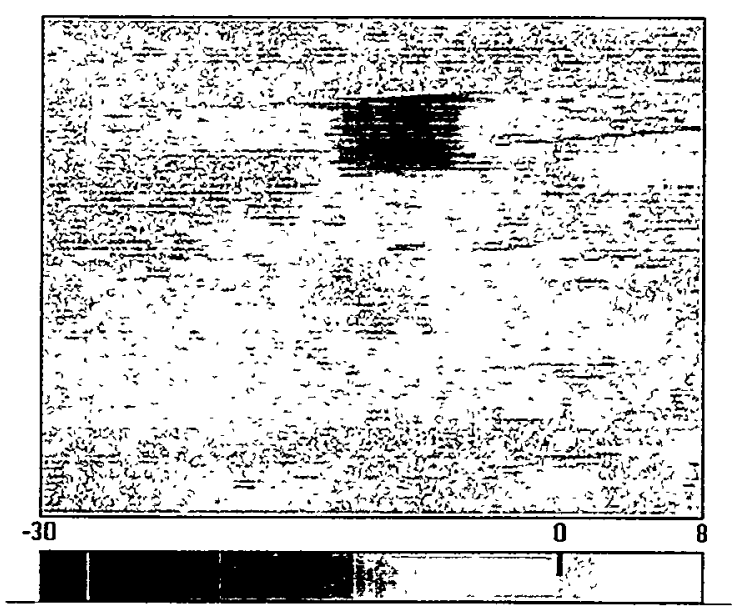

(b) $S_{1}$

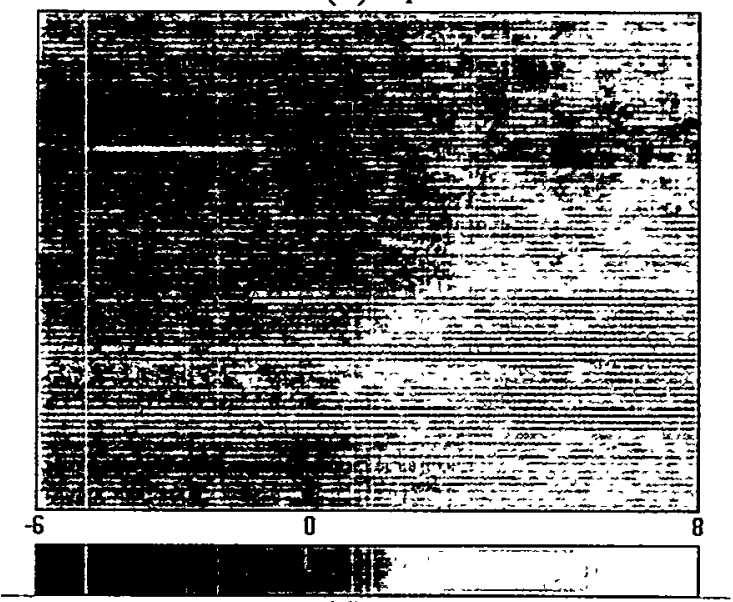

(d) $S_{3}$

Figure 19. Stokes-parameter images of a Cessna aircraft. Note the strong emission polarization signature in the $S_{1}$ image. The images have been independently scaled and offset for contrast, as indicated by the scale below each image. 


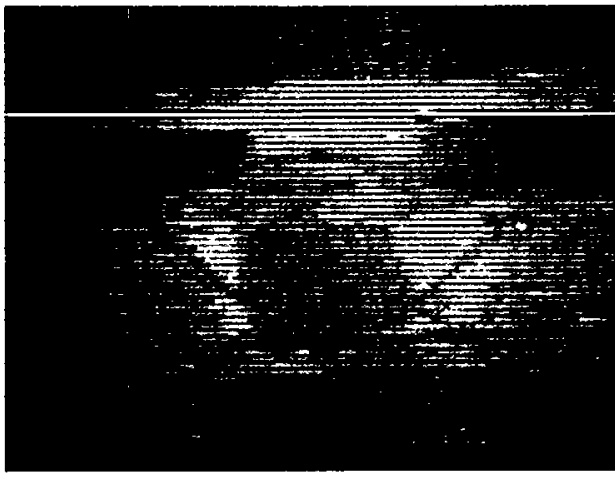

(a)

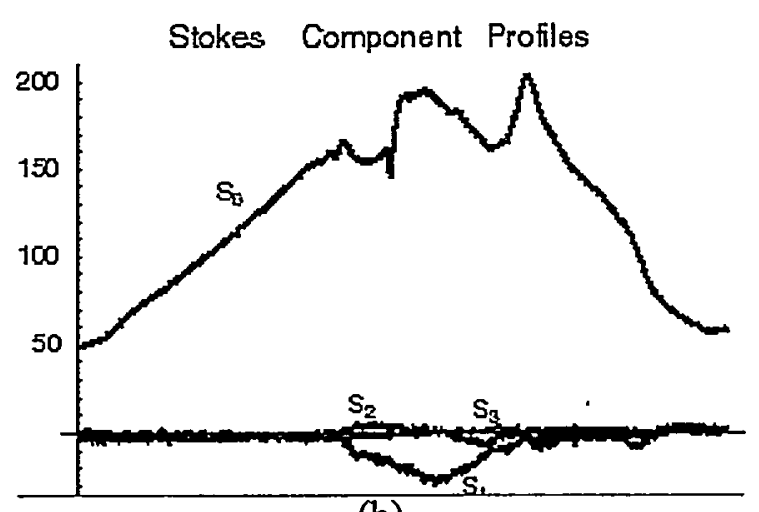

(b)

Figure 20. Location of profile through aircraft image (Figure 19) and profiles corresponding to each of the four Stokes parameters. See text for discussion.

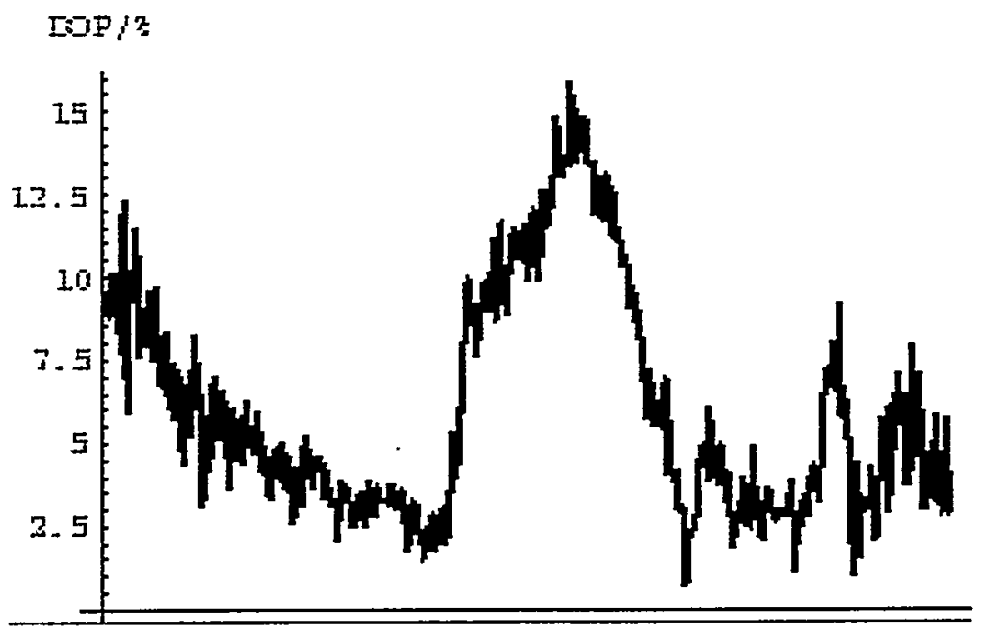

Figure 21. The DOP for the profile indicated in Figure 20(a). The horizontal axis represents pixels along the profile in the aircraft image.

The magnitude of the circular-polarization Stokes parameter, $S_{3}$, in the case of aircraft data was again comparable to the noise in the calculated Stokes-vector components.

\section{CONCLUSIONS AND FUTURE WORK}

We constructed an optimized imaging polarimeter based on a PtSi MWIR camera, a fixed wire-grid polarizer and a form-birefringent retarder. This imaging polarimeter was used in outdoor experiments that involved natural and manmade objects. We recorded a maximum degree of polarization of $49 \%$ associated with the hood of an idling automobile.

A significant enhancement of the imaging polarimeter described in this report would result from using an infrared camera with higher bit resolution, e.g. 12 bits rather than the 8 bits as was the case with the PtSi camera. We estimate that in the case of the PtSi camera, approximately two bits were "lost" to a background-signal pedestal. The background-signal pedestal arose from the imaging-polarimeter optics, i.e., the lens and the rotating retarder. 
We estimated the magnitude of the background-signal pedestal by placing a liquid-nitrogen cooled sponge in front of the imaging polarimeter. Any replacement camera system should retain the capability of the current system of cryogenically cooling emissive optical elements (such as wire grid polarizers and filters).

The Stokes parameter $S_{3}$ is a weak component in the scenes that we have imaged. Elimination of the capability to measure $S_{3}$ considerably simplifies polarimeter design. Such simplification is especially valuable when one considers techniques for snapshot imaging polarimetry.

The ability to simultaneously collect all measurements necessary to retrieve Stokes parameters eliminates the problems of target motion, changes in irradiance conditions, e.g., clouds, and drifts in offset and responsivity of the infrared camera.

Promising solutions include an imaging array sensor with pixels that are each coated with a differently-oriented linear polarizer. This solution is similar to a color imaging array in that spectral filters are now replaced by polarizing elements. A disadvantage of this solution is that a surface with the same Stokes-parameter signature must fill multiple instantaneous fields of view in order to yield a complete set of data. ${ }^{7}$

Another promising solution is the wedged double Wollaston prism (see Figure 22). ${ }^{8}$ Such a prism can be used to simultaneously create four images of the field of view on one imaging array (see Figure 23). A disadvantage of this solution is that the four simultaneous images shown in Figure 23 are not necessarily identically aligned with the grid of detector elements on the imaging array. Consequently, some form of resampling will be required before transforming the raw data into the Stokes parameters $S_{0}, S_{1}$, and $S_{2}$. A second disadvantage stems from the necessarily rectangular field of view that results from the use of a focal plane array with a nearly square format, e.g., 512 by 512 detectors. Figure 23 illustrates this constraint in the case of imaging a military truck. First, light in the pupil where the wedged double Wollaston is located is split by a pair of wedge prisms. At this point, light is deviated independently of polarization. This is the first image doubling illustrated in Figure 23. Next, two Wollaston prisms are used to accomplish a second division of light in the pupil. This time, light is deviated in different directions according to polarization. The four resultant images correspond to four different states of polarization that are indicated by arrows in Figure 23. 


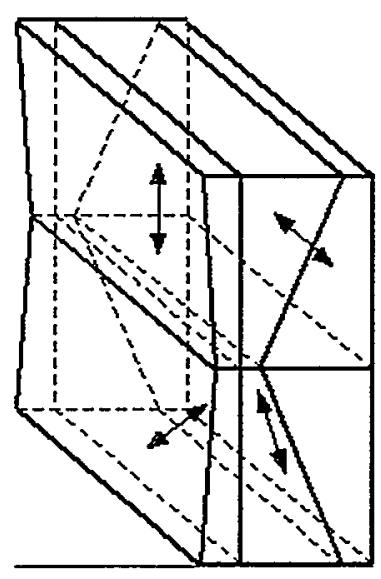

Figure 22. Wedged double Wollaston prism. Illustration adapted from Ref. 8 . The prism construction is shown with the fast axes of the birefringent segments of the wedged double prism indicated by double-ended arrows.

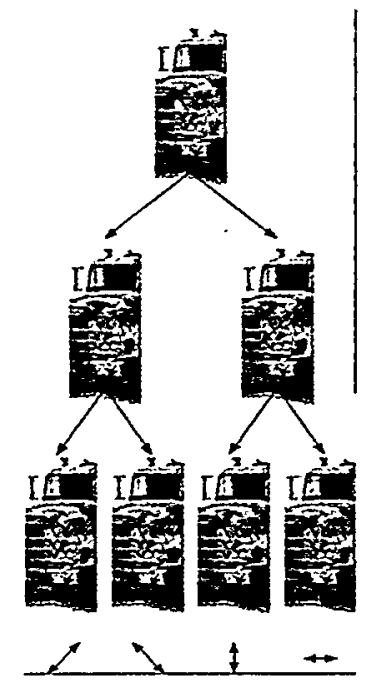

Figure 23. The sequence in which the four polarization images are created on one focal plane by means of the wedged double Wollaston prism. See text for details. 


\section{APPENDIX A. POINCARÉ SPHERE CUTOUT.}

To assemble the Poincaré sphere model, begin by cutting out the leaf-shaped pieces of the following pattern. Assemble the leaves into a flower-like pattern with the numbered ends together, using a small (c. $1 \mathrm{~cm}$ on a side) piece of cellophane tape on the back to hold them together (see Figure A-1, part a). Use the numbers on the ends of the leaves to ensure they are in the right order (like numbers should be adjacent). Then flex the leaves to join the other ends, securing them with a second piece of tape on the inside of the sphere (Figure A-1, part b). Figure A-1, part c shows the finished model.

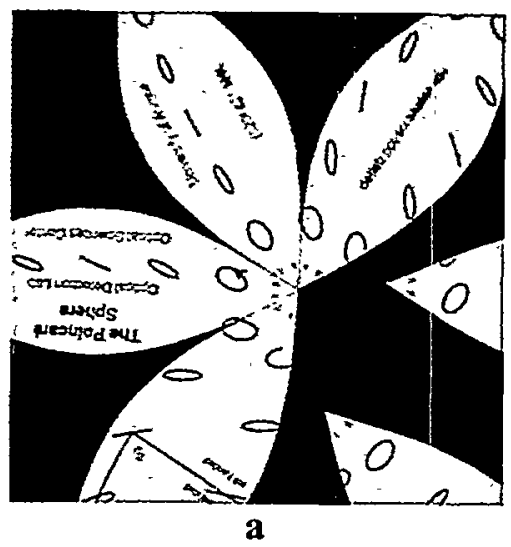

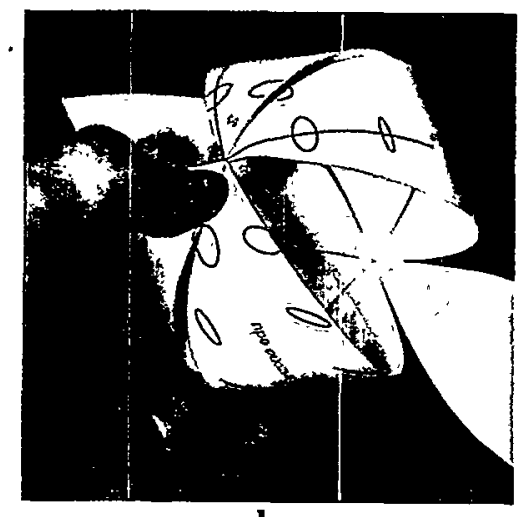

b

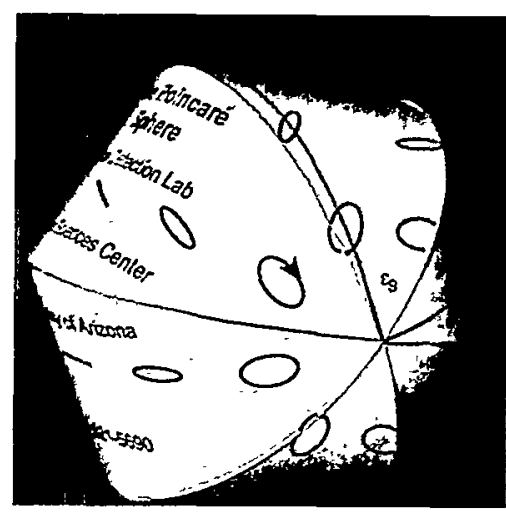

c

Figure A-1: Steps in assembling the Poincaré sphere model. 
Intentionally Left Blank 

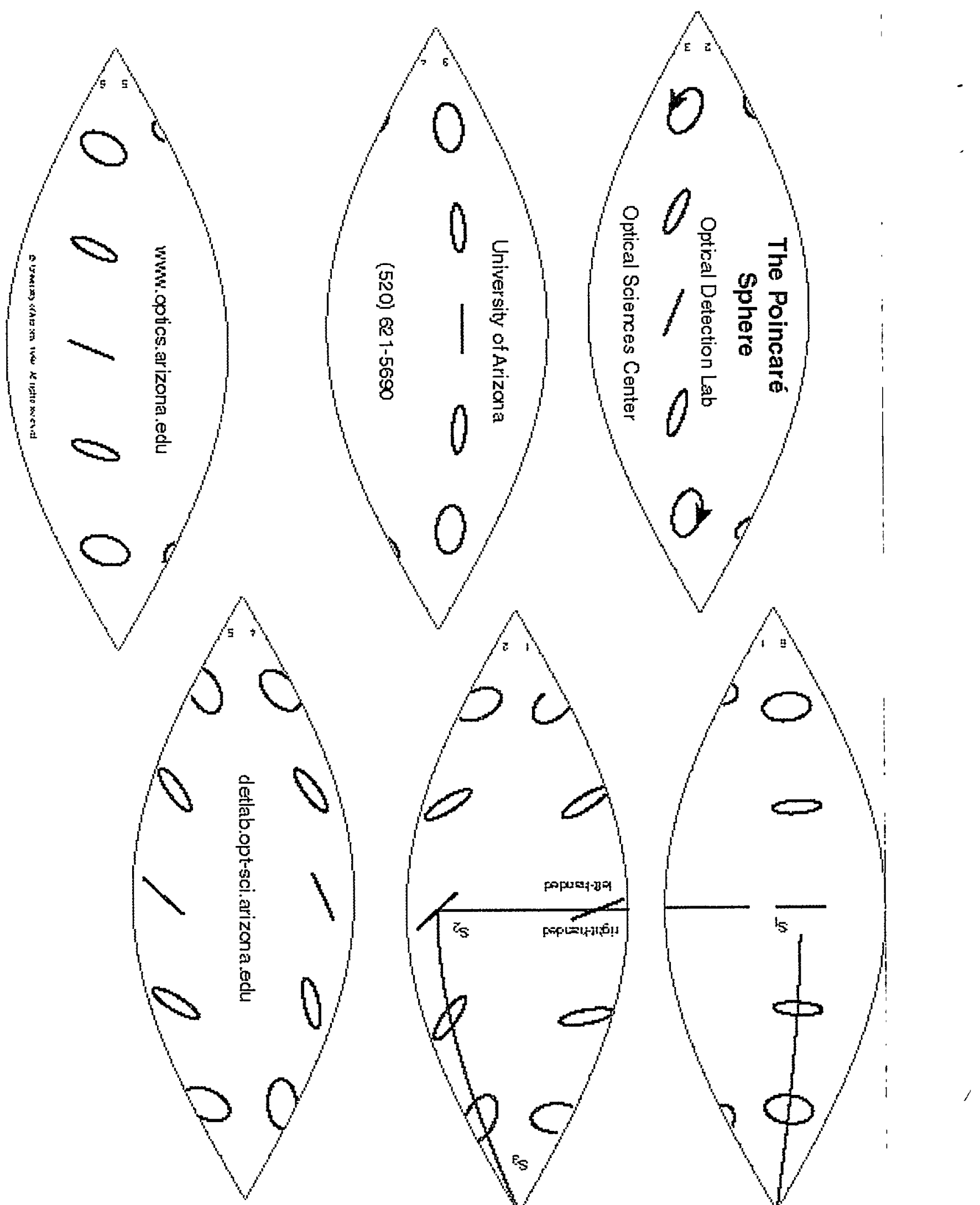
APPENDIX B. VIRTUAL INSTRUMENT PROGRAM TO CONTROL THE CHARACTERIZATION SET-UP

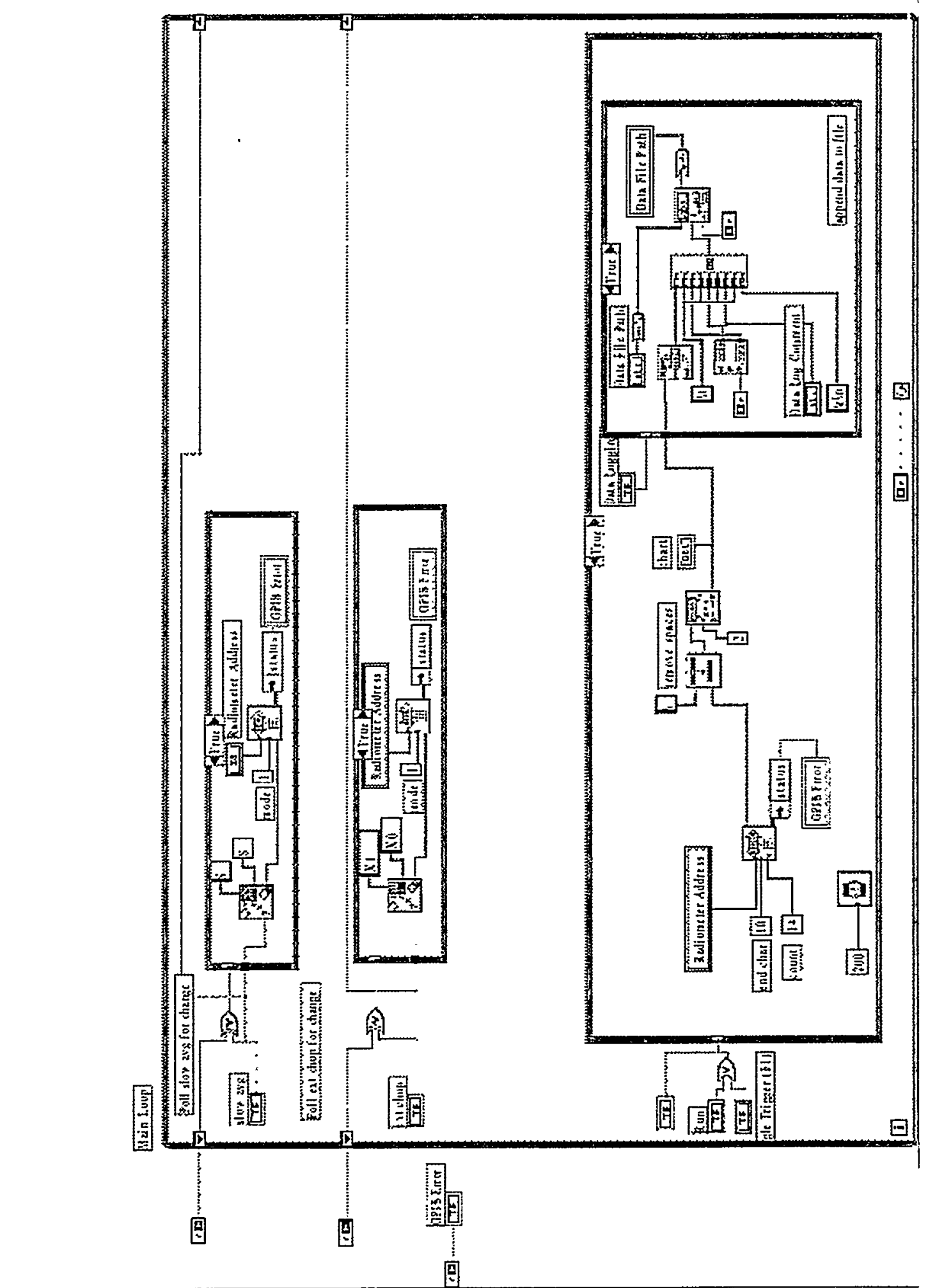

Note that the FALSE branches of all IF blocks are empty. 


\section{REFERENCES}

${ }^{1}$ A. Ambirajan and D.C. Look, Jr., "Optimum angles for a polarimeter: Part I," Opt. Eng., 34, No. 6, pp. 1651-1655 (June 1995).

${ }^{2}$ A. Ambirajan and D.C. Look, Jr., "Optimum angles for a polarimeter: Part II," Opt. Eng., 34, No. 6, pp. 1656-1658 (June 1995).

${ }^{3}$ M. Born and E. Wolf, Principles of Optics, $6^{\text {th }}$ ed., p. 30, Pergamon, Oxford, 1980.

${ }^{4}$ R.M.A. Azzam, N.M. Bashara, Ellipsometry and Polarized Light, Elsevier Science, Amsterdam, 1977.

${ }^{5}$ W.J. Tropf, et al., "Properties of Crystals and Glasses," in Handbook of Optics, Ch. 33 (1996).

${ }^{6}$ M. Born, Optik; ein Lehrbuch der Elektromagnetischen Lichttheorie, p. 31, Verlag von Julius Springer, Berlin, 1933.

${ }^{7}$ G.P. Nordin, J.T. Meier, P.C. Deguzman, and M.W. Jones, "Micropolarizer array for infrared imaging polarimetry," Journal of the Optical Society of America A, 16, No.5, p.1168-74 (May 1999).

${ }^{8}$ E. Oliva, "Wedged double Wollaston, a device for single shot polarimetric measurements," Astron. Astrophys. Suppl. Ser. 123, 589-592 (1997). 


\section{DISTRIBUTION:}

1 M. R. Descour University of Arizona Optical Sciences Center Tucson, AZ 85773

1 D. S. Sabatke University of Arizona Optical Sciences Center Tucson, AZ 85773

1 J. P. Garcia

University of Arizona Optical Sciences Center Tucson, AZ 85773

1 E. L. Dereniak University of Arizona Optical Sciences Center Tucson, AZ 85773

1 John Lamonica Farmers Investment Co. Sahuarita, AZ 85629

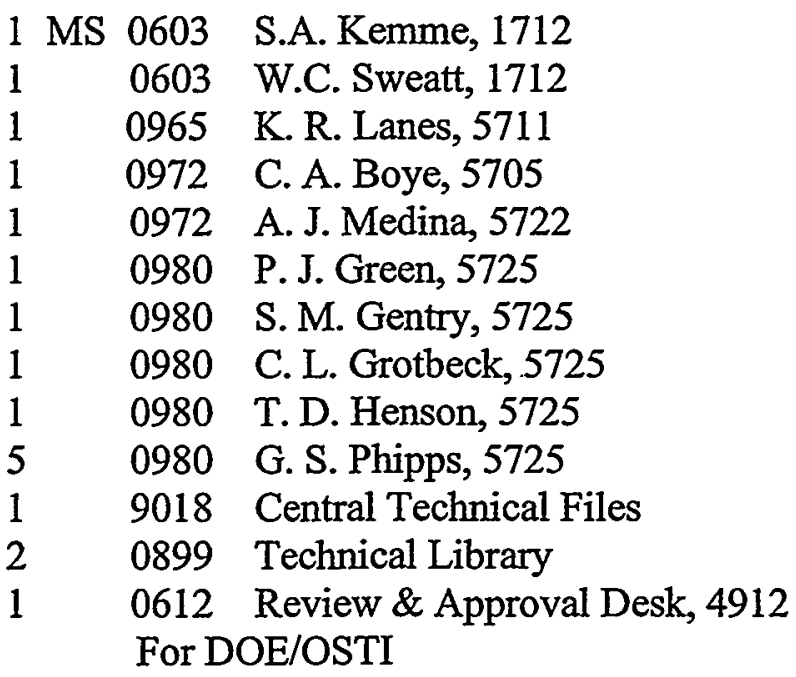

